Article

\title{
An Engineered Biliverdin-Compatible Cyanobacteriochrome Enables a Unique Ultrafast Reversible Photoswitching Pathway
}

\author{
Sean R. Tachibana ${ }^{1} \oplus$, Longteng Tang ${ }^{1}{ }^{\oplus}$, Liangdong Zhu ${ }^{1} \oplus$, Yuka Takeda ${ }^{2}$, Keiji Fushimi ${ }^{2, \dagger}$,

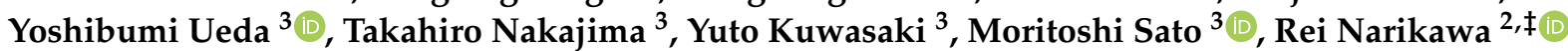 \\ and Chong Fang $1, * \mathbb{B}$
}

Citation: Tachibana, S.R.; Tang, L.; Zhu, L.; Takeda, Y.; Fushimi, K.; Ueda, Y.; Nakajima, T.; Kuwasaki, Y.; Sato,

M.; Narikawa, R.; et al. An Engineered Biliverdin-Compatible Cyanobacteriochrome Enables a Unique Ultrafast Reversible Photoswitching Pathway. Int. J. Mol. Sci. 2021, 22, 5252. https://doi.org/ $10.3390 /$ ijms 22105252

Academic Editors: Taiha Joo and Cheol Ho Choi

Received: 16 April 2021

Accepted: 14 May 2021

Published: 16 May 2021

Publisher's Note: MDPI stays neutral with regard to jurisdictional claims in published maps and institutional affiliations.

Copyright: (c) 2021 by the authors. Licensee MDPI, Basel, Switzerland. This article is an open access article distributed under the terms and conditions of the Creative Commons Attribution (CC BY) license (https:/ / creativecommons.org/licenses/by/ $4.0 /)$.
1 Department of Chemistry, Oregon State University, 153 Gilbert Hall, Corvallis, OR 97331-4003, USA; tachibas@oregonstate.edu (S.R.T.); tanglo@oregonstate.edu (L.T.); zhul@oregonstate.edu (L.Z.)

2 Graduate School of Integrated Science and Technology, Shizuoka University, Shizuoka 422-8529, Japan; 0830mon1201@gmail.com (Y.T.); fushimi.keiji@shizuoka.ac.jp (K.F.); narikawa.rei@shizuoka.ac.jp (R.N.)

3 Graduate School of Arts and Sciences, University of Tokyo, Tokyo 153-8902, Japan; yoshibumiueda@gmail.com (Y.U.); ctnaka@mail.ecc.u-tokyo.ac.jp (T.N.); kuwasakiyuto@g.ecc.u-tokyo.ac.jp (Y.K.); moritoshisato@g.ecc.u-tokyo.ac.jp (M.S.)

* Correspondence: Chong.Fang@oregonstate.edu; Tel.: +1-541-737-6704

+ Current address: Graduate School of Science, Innovation and Technology, Kobe University, Kobe 657-8501, Japan.

$\ddagger$ Current address: Department of Biological Sciences, Graduate School of Science, Tokyo Metropolitan University, Tokyo 192-0397, Japan.

\begin{abstract}
Cyanobacteriochromes (CBCRs) are promising optogenetic tools for their diverse absorption properties with a single compact cofactor-binding domain. We previously uncovered the ultrafast reversible photoswitching dynamics of a red/green photoreceptor AnPixJg2, which binds phycocyanobilin (PCB) that is unavailable in mammalian cells. Biliverdin (BV) is a mammalian cofactor with a similar structure to PCB but exhibits redder absorption. To improve the AnPixJg2 feasibility in mammalian applications, AnPixJg2_BV4 with only four mutations has been engineered to incorporate BV. Herein, we implemented femtosecond transient absorption (fs-TA) and ground state femtosecond stimulated Raman spectroscopy (GS-FSRS) to uncover transient electronic dynamics on molecular time scales and key structural motions responsible for the photoconversion of AnPixJg2_BV4 with PCB (Bpcb) and BV (Bbv) cofactors in comparison with the parent AnPixJg2 (Apcb). Bpcb adopts the same photoconversion scheme as Apcb, while BV4 mutations create a less bulky environment around the cofactor $\mathrm{D}$ ring that promotes a faster twist. The engineered Bbv employs a reversible clockwise/counterclockwise photoswitching that requires a two-step twist on $\sim 5$ and 35 picosecond (ps) time scales. The primary forward $\mathrm{P}_{\mathrm{fr}} \rightarrow \mathrm{P}_{\mathrm{o}}$ transition displays equal amplitude weights between the two processes before reaching a conical intersection. In contrast, the primary reverse $\mathrm{P}_{\mathrm{o}} \rightarrow \mathrm{P}_{\mathrm{fr}}$ transition shows a 2:1 weight ratio of the $\sim 35$ ps over 5 ps component, implying notable changes to the D-ring-twisting pathway. Moreover, we performed pre-resonance GS-FSRS and quantum calculations to identify the Bbv vibrational marker bands at $\sim 659,797$, and $1225 \mathrm{~cm}^{-1}$. These modes reveal a stronger H-bonding network around the BV cofactor A ring with BV4 mutations, corroborating the D-ring-dominant reversible photoswitching pathway in the excited state. Implementation of BV4 mutations in other PCB-binding GAF domains like AnPixJg4, AM1_1870g3, and NpF2164g5 could promote similar efficient reversible photoswitching for more directional bioimaging and optogenetic applications, and inspire other bioengineering advances.
\end{abstract}

Keywords: far-red/orange cyanobacteriochromes; structure-activity relationships; time-resolved spectroscopy; reversible photoswitching; optogenetics

\section{Introduction}

Phytochromes, bacteriophytochromes, and cyanobacteriochromes (CBCRs) are among a superfamily of linear tetrapyrrole (bilin chromophore that is metabolically derived from 
heme) binding photoswitching proteins [1-3]. In nature, these proteins act as photosensing and signaling units that allow organisms to detect and respond to light from the UV to near-IR range [4-8]. Previous efforts have engineered these proteins as both non-invasive optogenetic tools [9-11] and fluorescent tags in vivo [12,13]. CBCRs have shown to be a versatile tool in optogenetic applications by requiring only a single compact domain for incorporation of the cofactor chromophore, the photosensing unit that can be considered as the

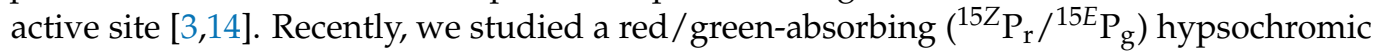
CBCR named AnPixJg2 using the wavelength-tunable femtosecond transient absorption (fs-TA) and ground-state femtosecond stimulated Raman spectroscopy (GS-FSRS) [15]. We uncovered the initial ultrafast dynamics as the phycocyanobilin (PCB cofactor in protein matrix) photoswitches from the $P_{g}$ to $P_{r}$ and $P_{r}$ to $P_{g}$ conformers in a reversible manner (controlled by a combination of actinic laser pump pulses and continuous-wave/cw LEDs). The nomenclature of $Z$ and $E$ refers to the $C-D$ ring methine bridge $C 15=C 16$ configuration in the cis and trans state, respectively. The $\mathrm{P}_{\mathrm{g}} \rightarrow \mathrm{P}_{\mathrm{r}}$ conversion exhibits a more downhill reaction with a $\sim 3$ ps lifetime to reach a conical intersection (CI) and relaxes into a Lumi-G state on the $\sim 30$ ps time scale. In contrast, the $\mathrm{P}_{\mathrm{r}} \rightarrow \mathrm{P}_{\mathrm{g}}$ conversion shows a longer-lived excited state with $\sim 13$ and 217 ps time constants to reach a $\mathrm{CI}$, which takes a much longer time to further relax in the ground state of Lumi-R and beyond.

Although AnPixJg2 has shown to be an efficient photoswitching protein, some major drawbacks involve the lack of PCB in mammalian cells and a green absorbing $\left({ }^{15 E} \mathrm{P}_{\mathrm{g}}\right)$ form outside the optimal biological window, which render it less ideal for mammalian applications. Many protein engineers have drawn inspirations from similar photoswitching proteins such as bacteriophytochromes, which incorporate the biliverdin (BV) cofactor that is intrinsic in mammalian cells. BV also red-shifts the absorption profiles of both conformational states due to a larger electronic conjugation than the PCB molecular framework [16]. Unlike CBCRs, bacteriophytochromes require three domains as a large photosensory core module with global structural preferences for BV incorporation and are typically used as fluorescent probes due to their higher fluorescence quantum yield [17]. In contrast, CBCRs that incorporate $\mathrm{PCB}$ as the cofactor exhibit low binding affinity for BV due to the double-bond carbon that cysteine (e.g., Cys321) covalently binds to (see Figure 1A-C). The different binding sites shift the cofactor location in the pocket, which decreases binding and protein expression [18].

To expand the functional space of CBCRs, the canonical AnPixJg2 has been engineered to accept the BV cofactor (AnPixJg2_BV4) with a solved crystal structure (PDB: 5ZOH) [18]. This advance was achieved by mutating four key residues around the cofactor pocket (H293Y, F308T, H318Y, and I336V). The BV incorporation red-shifts the red/green absorption to a far-red/orange absorption profile. The overall structure of AnPixJg2 with PCB cofactor (Apcb for short) and AnPixJg2_BV4 with BV cofactor (Bbv for short) was shown to be quite similar. The major structural difference was identified to be the BV cofactor shifted by $\sim 0.75 \AA$ due to the canonical Cys 321 covalently binding at the $C 3^{2}$ instead of $C 3^{1}$ in PCB (Figure 1A-C). Although shifted in Bbv, five crucial residues responsible for cofactor stability (W289, D291, R301, H322, and Y352) were also shown to be highly conserved in the cofactor vicinity. Of the four mutations (H293Y, F308T, H318Y, and I336V), F308T and I366V were found to be the most crucial for BV incorporation and expression. Both of these residues are located near cofactor $C$ and $D$ rings that reduce steric hindrance upon the BV-acceptable mutations. Besides achieving spatially compact residues, F308T and H318Y both contribute to better stabilize the $\mathrm{C}$ ring propionate group through $\mathrm{H}$-bonding. The same BV4 mutations were also demonstrated in several other PCB GAF domains with clearly improved BV-binding efficiencies [18]. 

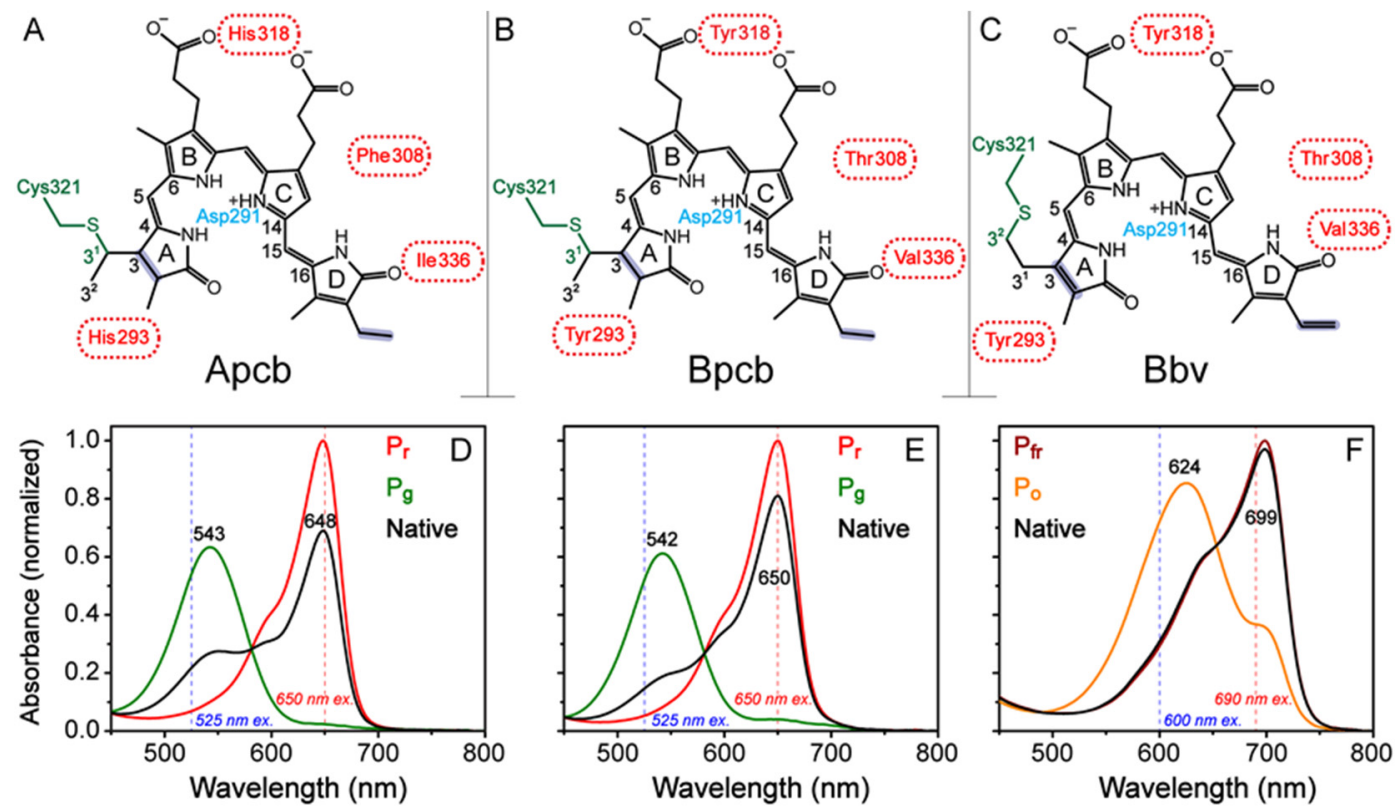

Figure 1. Representative chromophore and surrounding protein residues of (A) AnPixJg2_PCB, (B) AnPixJg2_BV4_PCB, and (C) AnPixJg2_BV4_BV. The associated native/dark-adapted states of ${ }^{15 Z_{\mathrm{P}}},{ }^{15 Z} \mathrm{P}_{\mathrm{r}}$, and ${ }^{15 Z} \mathrm{P}_{\mathrm{fr}}$ are depicted. Four residues responsible for the BV incorporation are shown (red) with Cys321 (green) that covalently binds cofactor to protein pocket. Key changes to cofactor conjugation are highlighted by purple shades with Asp291 (cyan) as an important H-bonding partner to A, B, and C rings. The absorption spectra of (D) Apcb, (E) Bpcb, and (F) Bbv are normalized to the reddestabsorbing species. Sample spectra were collected after ambient light, $505 \mathrm{~nm}$ LED, $650 \mathrm{~nm}$ LED, $600 \mathrm{~nm}$ LED, and a tungsten lamp with $650 \mathrm{~nm}$ longpass filter irradiation for $5 \mathrm{~min}$ for the native (black), $\mathrm{P}_{\mathrm{r}}$ (red), $\mathrm{P}_{\mathrm{g}}$ (green), $\mathrm{P}_{\text {fr }}\left(\right.$ dark red), and $\mathrm{P}_{\mathrm{O}}$ (orange) species, respectively. The actinic pump wavelengths for fs-TA experiments are labeled by vertical dashed blue and red lines in each panel.

Since internal conversion between the spin-allowed electronic states typically occurs on ultrafast time scales that precede fluorescence events on the nanosecond (ns) time scale [19], the tracking of Bbv cofactor ultrafast dynamics could uncover how the BV4 mutations affect the photoswitching pathways in real time. We implemented a systematic study using fs-TA with global analysis, and GS-FSRS with quantum calculations of AnPixJg2_BV4 with PCB cofactor (Bpcb for short), Apcb, and Bbv. These unique CBCR samples allow for a direct comparison of the same cofactor in different pockets (Apcb vs. $\mathrm{Bpcb})$, and different cofactors in the same pocket (Bpcb vs. Bbv). Correlating the effects of the BV4 mutations and BV cofactor on reversible photoswitching pathways with primary electronic and structural events in the non-equilibrium regime could facilitate future rational design of CBCRs, photoswitching proteins with cofactors, and other functional photochromic systems in general.

\section{Results and Discussions}

\subsection{Steady-State Electronic Spectroscopy with Altered Structural Contexts in CBCR Pockets}

In line with our previous study [15], a home-built LED box that fits four $5 \mathrm{~mm}$ throughhole LEDs was implemented to keep the samples in their respective conformers for all the spectroscopic experiments. LEDs with center wavelengths at 505, 600, and $650 \mathrm{~nm}$ were used to convert $C B C R$ s to the ${ }^{15 Z_{\mathrm{P}}},{ }^{15 Z_{\mathrm{P}}}$, and ${ }^{15 E} \mathrm{P}_{\mathrm{g}}$ conformers, respectively, and maintain those species in the sample reservoir during fs-TA and GS-FSRS experiments. To achieve conversion toward the ${ }^{15 E} \mathrm{P}_{\mathrm{o}}$ conformer of $\mathrm{Bbv}$, a tungsten lamp with a $650 \mathrm{~nm}$ longpass filter was used since our available LEDs did not convert the CBCR sample fast enough. The sample solution was then peristaltically pumped into a flow cell to prevent the LED irradiation from interfering with ultrafast spectroscopic measurements and also allow fresh sample solution to flow continuously, so refreshed samples always reach the laser irradiation spot for spectral data collection. For fs-TA experiments (see detailed results 
in Section 2.2 below), a 525 and $650 \mathrm{~nm}$ actinic pump ( 100 fs duration [20]) was used to excite the $\mathrm{P}_{\mathrm{g}}$ and $\mathrm{P}_{\mathrm{r}}$ conformers, respectively, of both Apcb and Bpcb (Figure 1D,E). For $\mathrm{Bbv}$, due to the red-shifted ground state absorption profile, a 600 and $690 \mathrm{~nm}$ actinic pump ( 100 fs duration) was implemented to excite the $\mathrm{P}_{\mathrm{o}}$ and $\mathrm{P}_{\mathrm{fr}}$ conformers (15E and $15 \mathrm{Z}$ isomers), respectively (Figure $1 \mathrm{~F}$ ).

The steady-state electronic absorption spectrum of the native CBCR conformer was the first spectrum collected and the sample in buffer solution was allowed to equilibrate under ambient light for $5 \mathrm{~min}$. Afterwards, the converted/target species was generated by specific light irradiation (see Table 1) for 5 min due to accumulated photoswitching from the starting species under a cw-light source [15]. Each spectrum was fitted using Gaussian peaks to identify major peak positions of $648 / 543,650 / 542$, and 699/624 nm for the Apcb $\mathrm{P}_{\mathrm{r}} / \mathrm{P}_{\mathrm{g}}, \mathrm{Bpcb} \mathrm{P}_{\mathrm{r}} / \mathrm{P}_{\mathrm{g}}$, and Bbv $\mathrm{P}_{\mathrm{fr}} / \mathrm{P}_{\mathrm{o}}$ conformers, respectively. Based on the spectra of native conformers, the $\mathrm{BV} 4$ mutations seem to promote a more homogeneous ground-state population as evidenced by the predominant $\mathrm{P}_{\mathrm{r}}$ and $\mathrm{P}_{\mathrm{fr}}$ forms of $\mathrm{Bpcb}$ and Bbv, respectively (Figure 1E,F, black traces). Upon comparison of the $\mathrm{P}_{\mathrm{r}}$ and $\mathrm{P}_{\mathrm{g}}$ absorption spectra of Apcb and Bpcb (Figure 1D,E, red and green traces), the BV4 mutations also cause a larger energy difference from $543 / 648 \mathrm{~nm}\left(\sim 2984 \mathrm{~cm}^{-1}\right)$ to $542 / 650 \mathrm{~nm}\left(\sim 3066 \mathrm{~cm}^{-1}\right)$. More importantly, the extended $\pi$-conjugation of Bbv cofactor molecular framework (Figure $1 \mathrm{C}$ ) improves its $\pi-\pi$ stacking with Trp289 [18] that contributes to the notably redder absorption of both $P_{o}$ and $\mathrm{P}_{\mathrm{fr}}$ states (Figure 1F, orange and dark red traces) than $\mathrm{P}_{\mathrm{g}}$ and $\mathrm{P}_{\mathrm{r}}$ states in Apcb as well as Bpcb (Figure 1D,E, green and red traces).

Table 1. Laser and LED irradiation conditions for the CBCR reversible photoswitching studies.

\begin{tabular}{cccccc}
\hline Sample & Initial State & LED (nm) & $\begin{array}{c}\text { Investigated State } \\
\text { Transition }\end{array}$ & $\begin{array}{c}\text { Fs-Actinic Pump } \\
\text { (nm) }\end{array}$ & $\begin{array}{c}\text { Optical Density } \\
\text { (OD/mm) }\end{array}$ \\
\hline Bpcb & $\mathrm{P}_{\mathrm{g}}$ & 650 & $\mathrm{P}_{\mathrm{g}} \rightarrow \mathrm{P}_{\mathrm{r}}$ & 525 & 0.8 \\
\hline $\mathrm{Bpcb}$ & $\mathrm{P}_{\mathrm{r}}$ & 505 & $\mathrm{P}_{\mathrm{r}} \rightarrow \mathrm{P}_{\mathrm{g}}$ & 650 & 0.8 \\
\hline $\mathrm{Bbv}$ & $\mathrm{P}_{\mathrm{o}}$ & 650 and above & $\mathrm{P}_{\mathrm{o}} \rightarrow \mathrm{P}_{\mathrm{fr}}$ & 600 & 0.43 \\
\hline $\mathrm{Bbv}$ & $\mathrm{P}_{\mathrm{fr}}$ & 600 & $\mathrm{P}_{\mathrm{fr}} \rightarrow \mathrm{P}_{\mathrm{o}}$ & 690 & 0.43 \\
\hline
\end{tabular}

${ }^{a}$ Measured at the reddest-absorbing peak of the thermally equilibrated/dark-adapted state (see Figure 1D-F). ${ }^{b}$ Generated by a tungsten lamp with a $650 \mathrm{~nm}$ longpass filter.

\subsection{Time-Resolved Electronic Spectroscopy Tracks Reversible Photoswitching of PCB and BV Cofactors in the AnPixJg2_BV4 Pocket}

The time-resolved electronic spectra of $\mathrm{CBCRs}$ in buffer solution using ultrafast laser pulses (Figure 2) track the cofactor molecular population as it evolves on the excited-state potential energy surface (PES). There are four characteristic features in the fs-TA spectra: excited-state absorption (ESA), hot ground-state absorption (HGSA), ground-state bleaching (GSB), and stimulated emission (SE). Both ESA and HGSA bands are positive whereas GSB and SE bands are negative. The GSB feature overlaps with the ground state absorption band (see Figure 1D-F) which distinguishes it from the typically red-shifted SE and HGSA features. Notably, the measured TA signal intensity for Bpcb photoswitching processes (ca. $\pm 18 \mathrm{mOD}, \mathrm{OD}$ is optical density, Figure $2 \mathrm{~A}, \mathrm{C}$ ) is similar to that for Apcb at a similar sample concentration ( $\mathrm{OD} \approx 0.8$ per $\mathrm{mm}$ at the $\mathrm{P}_{\mathrm{r}}$ absorption peak) [15], whereas the less concentrated $\mathrm{Bbv}\left(\mathrm{OD} \approx 0.4\right.$ per $\mathrm{mm}$ at the $\mathrm{P}_{\mathrm{fr}}$ absorption peak; see Table 1 in Section 2.1 above) displays much stronger TA intensity ( 2-2.5 fold, Figure 2B,D). This result implies a larger excited-state transition oscillator strength $[21,22]$ of the more conjugated BV cofactor than PCB cofactor in the CBCR pocket (see Figure 1) [18].

For corroboration, the raw experimental time-stacked fs-TA spectra (Supplementary Figure S1) exhibit the ultrafast dynamics of key electronic features, and the probedependent signal intensity ( $\pm 5 \mathrm{~nm}$ of peak wavelength, see Supplementary Figure S2) least-squares fits yield multiple exponentials with characteristic time constants and amplitude weights [23]. Since the protein cofactor photoswitching process typically involves excited state intermediates undergoing parallel and/or sequential transitions, the observed 
broad spectral features could be highly overlapped so the retrieved time constants from the probe-dependent fits may be convoluted or combined into an averaged value. Therefore, it is imperative to analyze and compare the least-squares fits with the associated time constants and amplitude weights for multiple TA marker bands to identify common features for the excited-state chromophores (see each panel in Supplementary Figure S2 for a specific photoswitching process in various $C B C R$ samples under study).
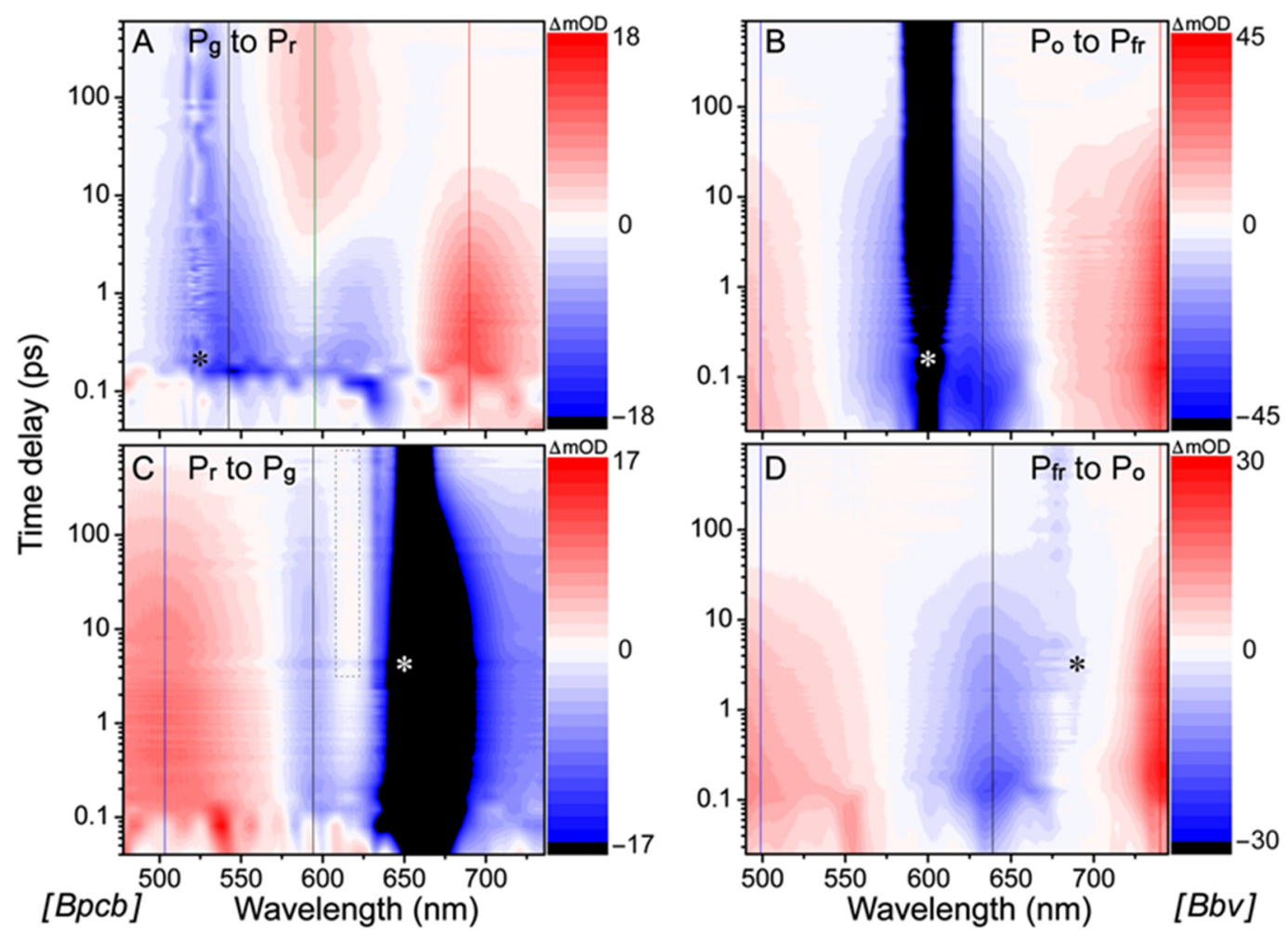

Figure 2. Time-resolved electronic spectra during reversible photoswitching of $\mathrm{Bpcb}$ and $\mathrm{Bbv}$ in buffer solution. The fs-TA 2D-contour plots of (A) Bpcb $\mathrm{P}_{\mathrm{g}} \rightarrow \mathrm{P}_{\mathrm{r}}$, (C) Bpcb $\mathrm{P}_{\mathrm{r}} \rightarrow \mathrm{P}_{\mathrm{g}}$, (B) Bbv $\mathrm{P}_{\mathrm{o}} \rightarrow \mathrm{P}_{\mathrm{fr}}$, and (D) Bbv $\mathrm{P}_{\text {fr }} \rightarrow \mathrm{P}_{\mathrm{o}}$ transitions were collected using a $525 \mathrm{~nm}$ actinic pump with $650 \mathrm{~nm}$ LEDs, $650 \mathrm{~nm}$ actinic pump with $505 \mathrm{~nm}$ LEDs, $600 \mathrm{~nm}$ actinic pump with $650 \mathrm{~nm}$ longpass-filtered tungsten lamp, and $690 \mathrm{~nm}$ actinic pump with $600 \mathrm{~nm}$ LEDs, respectively, up to 900 ps after electronic excitation. Strong scattering from the pump beam (specific location marked by the asterisk in each panel) was largely removed by subtracting the -2 ps TA trace from the subsequent traces, although residual scattering can be clearly seen in black (panels B and C). A weak positive band (light red color) is marked by the dashed rectangle at late times in panel (C). Key probe regions for dynamic analysis of the TA marker bands are highlighted by the color-coded vertical lines in each panel (see the associated least-squares fits with retrieved time constants in Supplementary Figure S2).

To help deconvolute the underlying cofactor species with pertinent time constants, global analysis was implemented to analyze TA data within the entire detection spectral and time window, allowing a holistic representation of the excited state electronic dynamics. The global analysis method typically employs two different models, evolution-associated difference spectrum (EADS) and decay-associated difference spectrum (DADS) [24]. In short, EADS uses multiple sequential states to model the spectra whereas DADS uses a parallel model with each species decaying with distinct lifetimes, which could provide complementary information for the complex TA data with overlapping spectral bands. The -2 ps (time delay, meaning that the probe pulse precedes the actinic pump pulse by 2 ps, hence serving as a useful background) TA spectrum was subtracted from the subsequent data traces to remove the strong scattering signal (some residuals can still be seen in Figure 2). During global analysis in Glotaran [25], the pump scattering was either set to zero if the feature was in the middle of the window or the window was trimmed when the scattering was at the edge so it would not interfere with the least-squares fits 
of the chromophore spectral features (Figure 3). Notably, the retrieved components from global analysis do not necessarily correspond to distinct molecular species, but rather, they represent a mathematical description of the spectral data with characteristic separable components and time constants in capturing the chromophore electronic dynamics.

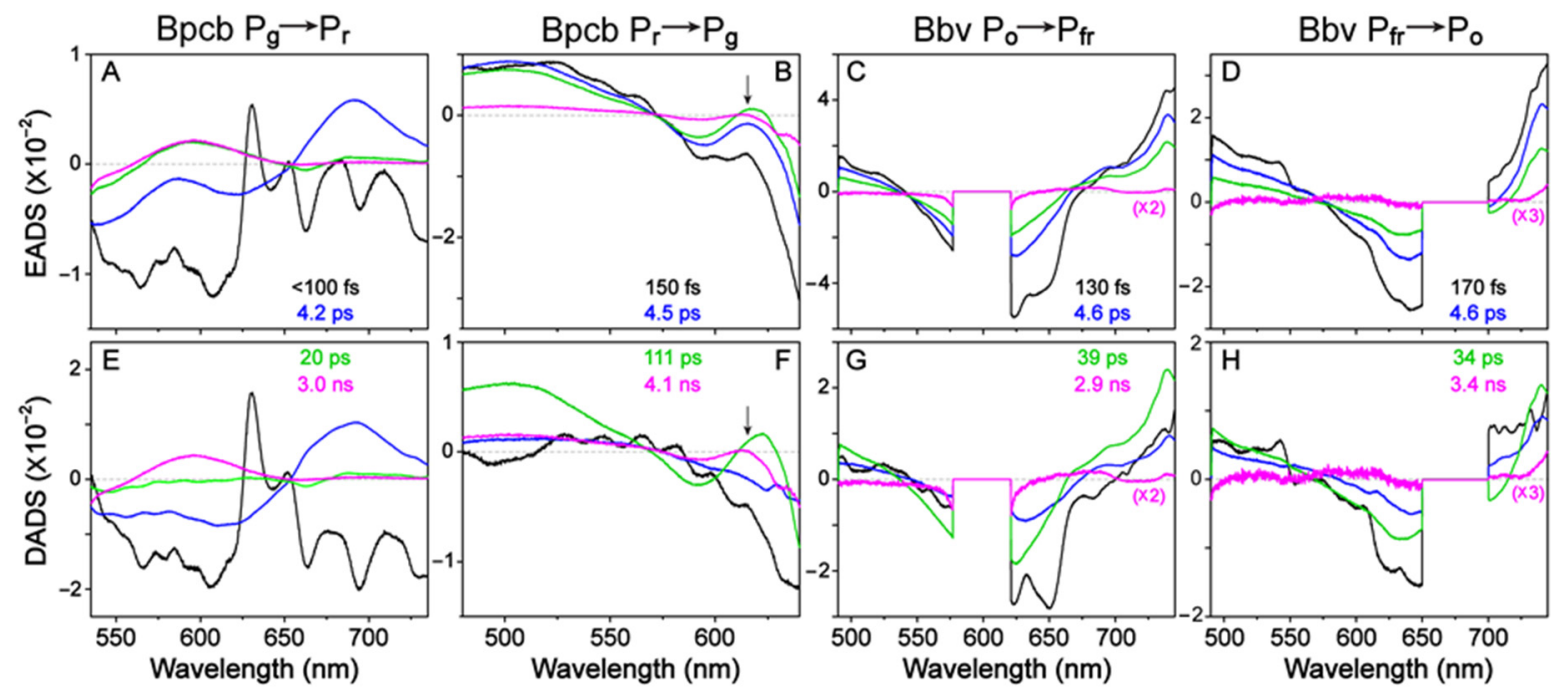

Figure 3. Global analysis of the fs-TA spectra during CBCR photoswitching processes. The underlying EADS/DADS of Bpcb $\mathrm{P}_{\mathrm{g}} \rightarrow \mathrm{P}_{\mathrm{r}}, \mathrm{Bpcb} \mathrm{P}_{\mathrm{r}} \rightarrow \mathrm{P}_{\mathrm{g}}$, Bbv $\mathrm{P}_{\mathrm{o}} \rightarrow \mathrm{P}_{\mathrm{fr}}$, and Bbv $\mathrm{P}_{\mathrm{fr}} \rightarrow \mathrm{P}_{\mathrm{o}}$ transitions are shown in panels $(\mathbf{A}, \mathbf{E}),(\mathbf{B}, \mathbf{F}),(\mathbf{C}, \mathbf{G})$, and $(\mathbf{D}, \mathbf{H})$, respectively. The retrieved spectra are ordered from fastest to slowest lifetimes in black, blue, green to magenta traces, with the associated lifetimes color-coded and listed in insets. To remove pump scattering effect on data analysis, the probe window was truncated when the scattering signal was on the edge of detection window (panels $(\mathbf{A}, \mathbf{B}, \mathbf{E}, \mathbf{F})$ ) or scattering region was set to zero (panels $(\mathbf{C}, \mathbf{D}, \mathbf{G}, \mathbf{H})$ ). Black arrows in panels $(\mathbf{B}, \mathbf{F})$ show a weak ESA band at $\sim 615 \mathrm{~nm}$.

The fs-TA spectral 2D-contour plot (Figure 2A) and global analysis results (Figure 3A,E) of Bpcb $\mathrm{P}_{\mathrm{g}} \rightarrow \mathrm{P}_{\mathrm{r}}$ photoconversion exhibit three major features that appear at the photoexcitation time zero: $\sim 542 \mathrm{~nm}$ GSB (matching the steady-state absorption peak of $\mathrm{P}_{\mathrm{g}}$ state in Figure 1E), $620 \mathrm{~nm}$ SE, and $690 \mathrm{~nm}$ ESA. These features correspond to the $\mathrm{P}_{\mathrm{g}}{ }^{*}$ (the asterisk denotes excited state) population. There is also rise of a positive feature around $595 \mathrm{~nm}$ that persists beyond the typical excited-state lifetime, hence it was assigned as an HGSA band of Lumi-G [15]. The $542 \mathrm{~nm}$ GSB band shows recovery dynamics of $\sim 350$ fs $(10 \%), 3.5$ ps $(45 \%), 31$ ps $(29 \%)$, and $1 \mu$ s (16\%) (Supplementary Figure S2A, black trace). The representative $1 \mu$ s component was used to model the long-lived population that does not return to original ground state within our detection time window. The most pronounced $690 \mathrm{~nm}$ ESA band shows clear decay dynamics with $400 \mathrm{fs}(9 \%), 4 \mathrm{ps}$ $(68 \%)$, and 27 ps (23\%) time constants (Supplementary Figure S2A, red trace), which is accompanied by a clear rise of the $595 \mathrm{~nm}$ HGSA that has been attributed to the Lumi-G species of PCB cofactor in the "hot" electronic ground state [15]. Due to spectral overlap in the visible spectral region, the probe-dependent fit of the $595 \mathrm{~nm}$ band yields a $200 \mathrm{fs}$ $(25 \%)$ and $3.4 \mathrm{ps}(37 \%)$ decay of the initial negative feature (Supplementary Figure S2A, green trace, reminiscent of the adjacent GSB band dynamics) and a $21 \mathrm{ps}(11 \%)$ rise of the Lumi-G HGS species that becomes apparent after $\sim 4$ ps (see the summary PES scheme below). The $\sim 1 \mu \mathrm{s}(27 \%)$ decay component was necessary for the fit since the long-lived signal eventually diminishes well beyond our current detection time window (see Methods in Section 3.3 below; a good separation of time constants allows the retrieval of longer time constants) [15].

Global analysis of the TA spectra for the photoinduced Bpcb $\mathrm{P}_{\mathrm{g}} \rightarrow \mathrm{P}_{\mathrm{r}}$ conversion requires five components to achieve a satisfactory fit, wherein the initial component fits a large coherent artifact with $<5$ fs lifetime (much lower than our cross-correlation time of $\sim 120 \mathrm{fs}[15,26])$ so it was removed from the figure to focus on major electronic dynamics of the protein cofactor (see Figure $3 \mathrm{~A}, \mathrm{E}$ ). The retrieved lifetimes were $<100 \mathrm{fs}, 4.2 \mathrm{ps}$, 
20 ps, and 3 ns that largely match the probe-dependent fits (Supplementary Figure S2A). The sub-ps time constant is known to be Franck-Condon (FC) relaxation as the photoexcited wavepacket moves across the PES after electronic excitation $[15,19,27,28]$. This ultrafast step can be seen as clear and distinct features emerge in EADS (Figure 3A) and DADS (Figure 3E, black to blue trace). The 4.2 ps component likely tracks a partial twisting motion of the D ring in $\mathrm{P}_{\mathrm{g}}{ }^{*}$ as it moves toward a CI, consistent with literature and the well-accepted D-ring twist along the photoswitching coordinate $[15,29]$. This interpretation is also supported by the loss of $\mathrm{P}_{\mathrm{g}}{ }^{*} \mathrm{ESA}$ and SE bands at $\sim 690$ and $620 \mathrm{~nm}$ (Figure 3A,E, blue to green trace), and the associated large amplitude weight that suggests a dominant relaxation pathway to the ground state. After the CI passage, the population diverges into two pathways. First, the twisted $\mathrm{P}_{\mathrm{g}}{ }^{*}$ can continue to twist and reach the Lumi-G state as the primary photoproduct species, which is evident by the $\sim 21 \mathrm{ps}$ rise of the Lumi-G HGSA band (Figure 3A,E, green to magenta trace; Supplementary Figure S2A, green trace). Second, the twisted $\mathrm{P}_{\mathrm{g}}{ }^{*}$ can reverse-twist back to $\mathrm{P}_{\mathrm{g}}$ in the ground state as indicated by the $\sim 31$ ps GSB band recovery (Supplementary Figure S2A, black trace). The $\sim 3$ ns component from global analysis and $\sim 1 \mu \mathrm{s}$ component from the probe-dependent fits are representative of a weak fluorescence process and further protein-pocket-facilitated cofactor motions as Lumi-G fully converts to $P_{r}$ in the electronic ground state $[15,30]$.

The Bpcb $\mathrm{P}_{\mathrm{r}} \rightarrow \mathrm{P}_{\mathrm{g}}$ TA spectra (Figure 2C) and global analysis (Figure 3B,F) show clear $\mathrm{P}_{\mathrm{r}}{ }^{*}$ modes with an ESA band around $\sim 520 \mathrm{~nm}$ and two GSB bands at 594 and $650 \mathrm{~nm}$, with the latter two bands matching the main ground state absorption peak and its bluer vibronic shoulder (Figure 1E, red trace). The strong scattering from $650 \mathrm{~nm}$ actinic pump made the probe-dependent fits in that region unreliable (see black shade in Figure 2 and orange asterisk in Supplementary Figure S1C). We thus fit the cleaner $594 \mathrm{~nm}$ GSB band recovery dynamics with $\sim 150 \mathrm{fs}(34 \%), 4.7 \mathrm{ps}(20 \%), 118 \mathrm{ps}(30 \%)$, and $1 \mathrm{~ns}(16 \%)$ time constants (Supplementary Figure S2C, black trace). Meanwhile, the $503 \mathrm{~nm}$ ESA band exhibits similar intensity decay time constants of $\sim 4.8$ ps (13\%), 254 ps (57\%), and $2.5 \mathrm{~ns}$ $(6 \%)$, but also requires a 28 ps (24\%) component for a satisfactory fit (Supplementary Figure S2C, blue trace). The retrieval of two intermediate time constants (28 and $254 \mathrm{ps)} \mathrm{instead} \mathrm{of}$ one 118 ps time constant (from GSB recovery) or 111 ps (from global analysis of the spectra within the detection window, see below) could be due to more complex dynamics when higher-lying electronic excited states are involved (e.g., the $503 \mathrm{~nm}$ ESA band due to the $S_{1}$ $\rightarrow \mathrm{S}_{\mathrm{n}}$ transition) [20,31], although the general time scale of $\sim 110-120$ ps is consistent for the PCB cofactor excited-state relaxation along the $S_{1}$ PES.

Notably, there is a discernible rise and decay of a positive band around $\sim 615 \mathrm{~nm}$ (see Figure 2C, dashed rectangle box) which resembles the fs-TA data of Apcb [15] and could be attributed to a slightly twisted excited-state intermediate $\left(\mathrm{P}_{\mathrm{r}}{ }^{* \prime}\right)$. To reduce the pump scattering effect and confirm the delayed onset of this specific ESA band, we performed a control experiment using a dilute $\mathrm{Bpcb}$ sample $\left(\mathrm{OD} \approx 0.2 / \mathrm{mm}\right.$ at the $\mathrm{P}_{\mathrm{r}}$ state absorption peak, about a quarter of the Bpcb sample concentration listed in Table 1 and presented in Figure $2 \mathrm{C}$ ) and observed clear dynamics of a $\sim 618 \mathrm{~nm}$ band with a much reduced scattering signal to the red side (Supplementary Figure S3). The $743 \mathrm{~nm}$ SE band shows similar decay dynamics to the bluer GSB band at $594 \mathrm{~nm}$ (Supplementary Figure S2C). The overall cofactor excited-state decay dynamics of Bpcb are faster than Apcb (see Section 2.5 below for a detailed comparison), suggesting that smaller residues in the chromophore vicinity of AnPixJg2_BV4 mutant [18] allow for faster ring-twisting events (likely accompanied by local environment relaxation) of the PCB cofactor [32].

Global analysis of the Bpcb $\mathrm{P}_{\mathrm{r}} \rightarrow \mathrm{P}_{\mathrm{g}}$ TA spectra yields $\sim 150 \mathrm{fs}, 4.5 \mathrm{ps}, 111 \mathrm{ps}$, and 4.1 ns lifetimes. As discussed above, the $150 \mathrm{fs}$ component reflects FC dynamics of the $\mathrm{P}_{\mathrm{r}}{ }^{*}$ species while the 4.5 ps component tracks the $\mathrm{P}_{\mathrm{r}}{ }^{*} \rightarrow \mathrm{P}_{\mathrm{r}}{ }^{* \prime}$ transition. This key assignment was made previously in the Apcb $\mathrm{P}_{\mathrm{r}} \rightarrow \mathrm{P}_{\mathrm{g}}$ conversion by a pronounced rise and decay of the $616 \mathrm{~nm}$ ESA band of $\mathrm{P}_{\mathrm{r}}{ }^{* \prime}$ species [15]. However, the more significant overlap with a stronger GSB band (versus that in Apcb) makes it more difficult to reliably fit the weak 615 $n m P_{r}{ }^{* \prime}$ ESA band dynamics of Bpcb. Nevertheless, transient spectral signatures of the $P_{r}{ }^{* \prime}$ 
state is evident from global analysis (Figure 3B,F, blue to green trace, see the black arrow) and the pertinent $\sim 620 \mathrm{~nm}$ ESA band decays away on the $111 \mathrm{ps}$ time scale, which likely corresponds to the non-equilibrium molecular movement toward a peaked CI $[27,33]$ that can repopulate the original ground state and convert to the Lumi-R state $[15,29]$. Notably, the $\mathrm{CI}$ is also evident from the dominant loss of excited-state features with the $111 \mathrm{ps}$ lifetime (Figure 3B,F, green to magenta trace), while the longer-lived $4.1 \mathrm{~ns}$ component with an SE band redder than $\sim 670 \mathrm{~nm}$ (Figure $2 \mathrm{C}$, with a more pronounced SE peak at $\sim 743 \mathrm{~nm}$ in the dilute CBCR sample in Supplementary Figure S3) is likely owing to the fluorescence transition from $\mathrm{P}_{\mathrm{r}}{ }^{*}[15,29]$. Overall, the $\mathrm{P}_{\mathrm{r}} \rightarrow \mathrm{P}_{\mathrm{g}}$ conversion is less efficient than the $\mathrm{P}_{\mathrm{g}}$ $\rightarrow \mathrm{P}_{\mathrm{r}}$ conversion since the majority of excited-state species return to the original ground state $[15,29,34]$, in accord with the observed longer lifetimes and the lack of prominent new TA features emerging at late times after $650 \mathrm{~nm}$ photoexcitation (see Figures $2 \mathrm{C}$ and 3B).

For comparison, the fs-TA spectra of the newly engineered Bbv exhibit similar features for both the $P_{o} \rightarrow P_{\text {fr }}$ and $P_{\text {fr }} \rightarrow P_{o}$ transitions (Figure 2B,D), which differ from the PCB cases (Figure 2A,C). Although the observed TA bands are shifted in wavelength, they both exhibit a blue ESA band at the edge of our spectral window $\left(<500 \mathrm{~nm}\right.$ ), a GSB band in the middle region (around 633 and $639 \mathrm{~nm}$ for $\mathrm{P}_{\mathrm{o}} \rightarrow \mathrm{P}_{\mathrm{fr}}$ and $\mathrm{P}_{\mathrm{fr}}$ $\rightarrow \mathrm{P}_{\mathrm{o}}$ transitions, respectively, see Supplementary Figure S1B,D), and a strong red ESA band $(>740 \mathrm{~nm})$. An expanded spectral window to the bluer or redder probe regions is required to determine the exact locations for the blue and red ESA peaks; however, the increased similarity between the TA signatures of BV's $P_{f r}$ and $P_{o}$ states versus ${ }^{P C B}$ 's $P_{r}$ and $P_{g}$ states indicates the major effect of the intrinsic cofactor electronic structure, which likely causes the largely unchanged TA bands that are consistent with much less energy separation between the two conformers in BV (624 and $699 \mathrm{~nm}$ in Figure 1F, $\sim 1719 \mathrm{~cm}^{-1}$ ) versus PCB $\left(542\right.$ and $650 \mathrm{~nm}$ in Figure 1E, $\sim 3066 \mathrm{~cm}^{-1}$ ). These common TA features in Figure 2B,D mainly track the $\mathrm{P}_{\mathrm{o}}{ }^{*}$ and $\mathrm{P}_{\mathrm{fr}}{ }^{*}$ electronic dynamics because they appear at the photoexcitation time zero and decay away with no clear wavelength shifts. It is notable that the $\mathrm{P}_{\mathrm{fr}}$ GSB band around $690 \mathrm{~nm}$ overlaps with the pump scattering that is largely removed for TA analysis (Supplementary Figure S1D). Interestingly, all the least-squares fits of excited state dynamics show similar time constants for both conversions of the BV cofactor (Supplementary Figure S2B,D), which differs significantly from the PCB cofactor counterparts (Supplementary Figure S2A,C). For the $\mathrm{P}_{\mathrm{o}} \rightarrow \mathrm{P}_{\text {fr }}$ conversion, the probe-dependent fits expose $\sim 125 \mathrm{fs}(43 \%), 4.6 \mathrm{ps}(18 \%)$, and 32 ps (39\%) time constants for the 499 nm ESA band, 150 fs (48\%), 4.6 ps (17\%), $39 \mathrm{ps}(34 \%)$, and $2.9 \mathrm{~ns}(1 \%)$ time constants for the $633 \mathrm{~nm}$ GSB band, and $125 \mathrm{fs}(27 \%)$, 4.6 ps $(23 \%), 39$ ps $(49 \%)$, and $2.9 \mathrm{~ns}(1 \%)$ time constants for the $740 \mathrm{~nm}$ ESA band (see Supplementary Figure S2B). Similar dynamic components are apparent for these TA bands that report on the common excited-state decay pathways of a presumably homogeneous $\mathrm{P}_{\mathrm{o}}$ * population [35-37] as discussed in detail below.

For the $\mathrm{P}_{\mathrm{fr}} \rightarrow \mathrm{P}_{\mathrm{o}}$ conversion, the corresponding probe-dependent fits expose $\sim 125 \mathrm{fs}$ (35\%), 4.8 ps (27\%), 39 ps (37\%), and $3.4 \mathrm{~ns}(1 \%)$ time constants for the $499 \mathrm{~nm}$ ESA band, $\sim 129$ fs $(50 \%), 4.6 \mathrm{ps}(21 \%), 37 \mathrm{ps}(28 \%)$, and $3.4 \mathrm{~ns}(1 \%)$ for the $639 \mathrm{~nm}$ GSB band, and $\sim 130 \mathrm{fs}(31 \%), 4.9 \mathrm{ps}(31 \%), 36 \mathrm{ps}(34 \%)$, and $3 \mathrm{~ns}(4 \%)$ time constants for the $740 \mathrm{~nm}$ ESA band (see Supplementary Figure S2D), which reflect the common excited-state decay pathways of a presumably homogeneous $\mathrm{P}_{\mathrm{fr}}{ }^{*}$ population. Note that such a systematic analysis of independent measurements on reversible photoswitching processes of the same CBCR sample (e.g., Bbv with specific light irradiation conditions listed in Table 1 in Section 2.1 above) substantiates the robustness of the aforementioned time constants and amplitude weights for the underlying dynamic components retrieved after an actinic pump. As further corroboration, global analysis of the $P_{o} \rightarrow P_{\text {fr }}$ (Figure 3C,G) and $P_{\text {fr }} \rightarrow$ $\mathrm{P}_{\mathrm{o}}$ (Figure 3D,H) transitions also output similar time constants of $\sim 130$ and $170 \mathrm{fs}, 4.6$ and $4.6 \mathrm{ps}, 39$ and $34 \mathrm{ps}$, and 2.9 and $3.4 \mathrm{~ns}$, respectively. Similar to the $\mathrm{Bpcb} \mathrm{P}_{\mathrm{g}} \rightarrow \mathrm{P}_{\mathrm{r}}$ conversion case (Figure $3 \mathrm{~A}, \mathrm{E}$ ), the fit for a large coherent artifact was removed in the reversible photoswitching between $P_{o}$ and $P_{f r}$ states of Bbv to retrieve the intrinsic underlying time 
constants along the excited-state PES of the protein cofactor. Moreover, though the EADS show the blue trace (4.6 ps lifetime) decaying into green trace ( 35 ps lifetime, Figure $3 C, D)$, the corresponding DADS show the green trace intensity higher than blue trace in both ESA regions (below $550 \mathrm{~nm}$ and above $720 \mathrm{~nm}$, Figure 3G,H). This finding is indicative of some deviations from a simple kinetic model that the 4.6 ps component precedes the $35 \mathrm{ps}$ component (see below).

To confirm these key observations and results for the Bbv photoswitching mechanisms, we performed the following control experiments: (1) Fs-TA data sets were collected at least twice with the freshly prepared protein samples on different days in the laser lab to ensure reproducibility. (2) The steady-state absorption spectra of the $\mathrm{P}_{\mathrm{o}}$ and $\mathrm{P}_{\mathrm{fr}}$ conformers were taken before and after every TA experiment to confirm that the protein samples maintain their photoswitching capabilities. (3) Control TA experiments using $600 \mathrm{~nm}$ actinic pump with $600 \mathrm{~nm}$ LEDs (Supplementary Figure S4A) and $704 \mathrm{~nm}$ actinic pump with the $650 \mathrm{~nm}$ longpass-filtered tungsten lamp (Supplementary Figure S4B) were conducted to ensure that the TA data in Figure 2B,D were not just collecting the same conformer or mixed species. (4) A visual confirmation was achieved that the actinic pump could convert the protein samples by holding the sample cuvette in the beam path before it was focused [15]. (5) A redder actinic pump at $704 \mathrm{~nm}$ was used and similar TA features were observed for the $\mathrm{P}_{\mathrm{fr}} \rightarrow \mathrm{P}_{\mathrm{o}}$ transition (see Supplementary Figure S5 and Figure 2D). With all these strategic, useful, and interwoven control experiments substantiating the aforementioned results, the observed similar dynamics of the $\mathrm{P}_{\mathrm{o}} \rightarrow \mathrm{P}_{\mathrm{fr}}$ and $\mathrm{P}_{\mathrm{fr}} \rightarrow \mathrm{P}_{\mathrm{o}}$ transitions infer a very alike or even "purely" reversible excited-state pathway of the BV cofactor in AnPixJg2_BV4.

The major differences between the aforementioned reversible photoswitching dynamics are the amplitude weights of the two intermediate components retrieved on the ps time scale. The $\mathrm{P}_{\mathrm{o}} \rightarrow \mathrm{P}_{\mathrm{fr}}$ conversion (Supplementary Figure S2B) shows about half the weight for the $\sim 5$ ps component $(18 \%, 17 \%$, and $23 \%$ ) compared to the $\sim 35$ ps component $(39 \%$, $34 \%$, and $49 \%$ ) for the blue ESA, GSB, and red ESA bands, respectively. In contrast, for the corresponding TA marker bands, the $\mathrm{P}_{\mathrm{fr}} \rightarrow \mathrm{P}_{\mathrm{o}}$ conversion (Supplementary Figure S2D) shows approximately equal weights for the $\sim 5$ ps $(27 \%, 21 \%$, and $31 \%)$ and $\sim 35$ ps $(37 \%$, $28 \%$, and $34 \%$ ) components. This experimental finding poses two interesting questions. (1) Why does Bbv exhibit similar photoswitching dynamics in both directions whereas the Apcb and Bpcb $\mathrm{P}_{\mathrm{g}} \rightarrow \mathrm{P}_{\mathrm{r}}$ and $\mathrm{P}_{\mathrm{r}} \rightarrow \mathrm{P}_{\mathrm{g}}$ conversions show different excited-state pathways? (2) Why are the amplitude weights of two largely conserved intermediate ps time constants the major differentiating factor between the $\mathrm{Bbv} \mathrm{P}_{\mathrm{o}} \rightarrow \mathrm{P}_{\mathrm{fr}}$ and $\mathrm{P}_{\mathrm{fr}} \rightarrow \mathrm{P}_{\mathrm{o}}$ photoconversion?

\subsection{Altered Excited-State Dynamics Arise from an Interplay between PCB or BV Cofactor and} AnPixJg2 or AnPixJg2_BV4 on Ultrafast Time Scales

To answer these fundamental questions, we first examined the differences between PCB and BV cofactors in the AnPixJg2_BV4 pocket. The location-shifted BV cofactor in the BV4 pocket moves the A ring closer to its H-bonding partners Asp291 and Trp289 [18]. The distance between the cofactor A ring nitrogen atom and the Asp291 oxygen atom decreases from $~ 1.95 \AA$ in the Apcb $P_{r}$ state crystal structure (PDB ID: 3W2Z) [8] to $1.76 \AA$ in the Bbv $P_{\text {fr }}$ state crystal structure (PDB ID: $5 \mathrm{ZOH}$ at $1.60 \AA$ resolution) [18]. The distance between the cofactor A ring carbonyl oxygen and the Trp289 nitrogen is $\sim 0.21 \AA$ closer in Bbv $(2.76 \AA)$ than Apcb $(2.97 \AA)$. The closer H-bonding partners and the extra electron density in the A ring would effectively restrict it from large twisting motions, while a slight twist of the A ring has been reported as a component in the photoconversion process $[15,37,38]$. By better stabilizing the A ring inside the protein pocket, more of the photoconverting energy could be localized at the D ring with a reversible clockwise/counterclockwise twist (i.e., along the same isomerization pathway but in opposite directions) by reducing motions away from the D ring.

As the D ring twists, there are two dihedral angles that correlate with the conversion between $P_{f r}$ and $P_{o}$ conformers. The dihedral closer to the $\mathrm{D}$ ring (D-dihedral) likely causes a smaller twisting motion than the dihedral closer to the $C$ ring (C-dihedral, see Figure $1 \mathrm{~A}-\mathrm{C})$, since the $\mathrm{D}$-dihedral twist is a volume-conserving motion whereas the 
C-dihedral twist likely increases the volume [39-41]. Upon excitation of $P_{f r}$ to $P_{f r}{ }^{*}$, the conjugated double bonds acquire more single-bond character and vice versa $[33,42,43]$, in accord with previous studies showing that the $\mathrm{C} 15=\mathrm{C} 16$ bond (directly connecting to the $D$ ring) is responsible for the major twisting between the $15 Z / E$ conformers [44-46]. By analyzing both dihedral angles for the available Apcb $P_{\mathrm{r}}$ state and Bbv $P_{\mathrm{fr}}$ state crystal structures, the C-dihedral (CCCC) around the bridge single bond $\left(36.08^{\circ}\right.$ vs. $\left.51.12^{\circ}\right)$ is more twisted in $\mathrm{Bbv}$ whereas the D-dihedral (CCCN) around the bridge double bond $\left(26.96^{\circ}\right.$ vs. $0.83^{\circ}$ ) is more twisted in Apcb from the pyrrole ring plane $[8,15,18]$. The BV4 mutations, specifically the more critical F308T and I336V, help to accommodate the shifted cofactor around the D ring due to less bulky residues [18]. Notably, if we attribute the observed 4.6 and $34 \mathrm{ps}$ in the $\mathrm{Bbv} \mathrm{P}_{\mathrm{fr}} \rightarrow \mathrm{P}_{\mathrm{o}}$ photoconversion (Figure $3 \mathrm{D}$ ) to the small-scale D-dihedral and large-scale C-dihedral twisting motions, respectively, we could then expect a smaller and larger time constant from the cofactor TA dynamics in Apcb due to the aforementioned starting geometry. Indeed for the C-dihedral twist, we observed a 217 ps component for the Apcb $\mathrm{P}_{\mathrm{r}} \rightarrow \mathrm{P}_{\mathrm{g}}$ transition [15], and a 111 ps component for the Bpcb $\mathrm{P}_{\mathrm{r}} \rightarrow \mathrm{P}_{\mathrm{g}}$ transition (Figure $3 \mathrm{~B}$ ) likely owing to an intermediate geometry of the $\mathrm{D}$ ring in between the Apcb $\mathrm{P}_{\mathrm{r}}$ and $\mathrm{Bbv} \mathrm{P}_{\mathrm{fr}}$ conformers. In contrast, for the D-dihedral twist on the few ps time scale, it is less sensitive to the initial geometry alone mainly due to its close interaction with an adjacent Val336 or Ile336 (in Bbv, Bpcb or Apcb, see Figure 1A-C) that could affect the faster time constant (in preparation for the subsequent larger twist motion) [20,47].

It is likely that Bpcb also takes advantage of the extra space around the cofactor and adopts a slightly shifted orientation from Bbv. The larger area around the $\mathrm{D}$ ring also explains the overall faster photoswitching dynamics in Bpcb than Apcb [32]. The slightly slower $\sim 4$ ps component in Bpcb $\mathrm{P}_{\mathrm{g}} \rightarrow \mathrm{P}_{\mathrm{r}}$ than $\sim 3$ ps in Apcb $\mathrm{P}_{\mathrm{g}} \rightarrow \mathrm{P}_{\mathrm{r}}$ conversion [15] can be explained by the shifted PCB cofactor in the AnPixJg2_BV4 pocket which would bring the D ring closer to Val336 and somewhat hinder the initial smaller twist (i.e., D-dihedral). Although the smaller twisting motion becomes slower, the larger twist (i.e., C-dihedral) occurs faster in the $\mathrm{P}_{\mathrm{g}} \rightarrow \mathrm{P}_{\mathrm{r}}$ photoconversion of Bpcb ( 20 ps) than Apcb ( 30 ps), both retrieved from global analysis of the corresponding TA spectra after $525 \mathrm{~nm}$ photoexcitation. We note that the larger twist occurs in the electronic ground state past the CI, likely owing to the metastable $\mathrm{P}_{\mathrm{g}}{ }^{*}$ species that undergoes a more downhill and efficient process than the more "trapped" $\mathrm{P}_{\mathrm{r}}{ }^{*}$ species that requires both twisting processes in the electronic excited state $[15,29]$. Interestingly for the latter case, the $\mathrm{P}_{\mathrm{r}} \rightarrow \mathrm{P}_{\mathrm{g}}$ photoconversion of $\mathrm{Bpcb}$ ( 4.5 and $111 \mathrm{ps)}$ also occurs faster than Apcb ( 13 and $217 \mathrm{ps}$ ) involving presumably small and large ring-twisting motions of the protein cofactor, both retrieved from global analysis of the corresponding TA spectra after $650 \mathrm{~nm}$ photoexcitation [15].

For Bbv, because the photoswitching pathways are fully reversible with largely conserved time constants, the amplitude weights from the TA signal intensity fits become crucial in gaining mechanistic insights into the excited-state pathways. Since Bpcb does not exhibit such a reversible TA pattern during photoswitching, the observed "fully reversible" pathway seems to be unique to Bbv (i.e., both AnPixJg2_BV4 pocket and BV cofactor are needed). The probe-dependent least-squares fits implement a parallel model of multiple processes with a common time zero, although it does not mean that the fastest component occurs first. By inspecting the Apcb and Bpcb photoswitching scheme, the 3-4 and 20-30 ps components likely represent the aforementioned small and large twisting processes, respectively, although the detailed assignments to excited- or ground-state dynamics need to come from the TA spectral analysis. The amplitude weight of each time constant is representative of how dominant the associated pathway is for energy dissipation. Since the Bbv TA bands emerge around photoexcitation time zero and then decay, while the peak wavelength remains largely unshifted up to 900 ps (Figure 2B,D), the observed TA band intensity dynamics likely track the excited-state population change along the ring-twisting coordinates. Notably, a larger twist could cause a larger change of the electronic transition oscillator strength for the resultant conformation than that of a smaller twist, which may 
contribute to the apparent TA band intensity decay dynamics. We thus focus on the change of amplitude weight ratios instead of exact weights for ensuing analysis.

In the $\mathrm{P}_{\mathrm{fr}} \rightarrow \mathrm{P}_{\mathrm{o}}$ conversion of $\mathrm{Bbv}$, the equal weights of the $\sim 5$ ps and 35 ps components (Supplementary Figure S2D) suggest that a 5 ps smaller twisting motion would populate the $\mathrm{P}_{\mathrm{fr}}{ }^{* \prime}$ state before undergoing the 35 ps larger twisting motion en route to the CI. The less energy-consuming and more facile 5 ps twist could reduce the chances of other nonradiative pathways that compete with the 35 ps twist, while the initial small twist out of the more stabilized $\mathrm{P}_{\mathrm{fr}}$ state may be required to help the $\mathrm{D}$ ring move away from the adjacent Val336 to allow more room for the subsequent large twist in the excited state (Figure $1 \mathrm{C}$ ). In the $\mathrm{P}_{\mathrm{o}} \rightarrow \mathrm{P}_{\text {fr }}$ conversion of Bbv, an unexpected observation that the $\sim 35$ ps larger twist being twice as dominant as the 5 ps smaller twist implies that the larger twisting motion occurs first. The more energy-consuming and less facile 35 ps twist would bring the excited state closer to the ground state, allowing for more nonradiative processes to compete with the smaller twist. This would effectively cause the weight of the smaller twisting component to drop in comparison with the larger twist [20,21]. After the smaller twist, the remaining population could pass through a CI and continue to undergo cofactor ring twist and the surrounding protein pocket relaxation to reach the isomerized state (see Section 2.5 below and Graphical Abstract for illustration). We note that if we attributed the $\sim 5$ and 35 ps to inhomogeneous species of $\mathrm{P}_{\mathrm{O}}{ }^{*}$ undergoing excited-state energy relaxation in parallel, then it would be presumptuous to explain the same time constants from two inhomogeneous subpopulations of $\mathrm{P}_{\mathrm{fr}}{ }^{*}$ because the two conformer states have different cofactor conformations in their respective surrounding protein pockets upon electronic excitation $[8,38]$.

In both $\mathrm{Bbv}$ conversions, most of the population returns to the original ground state. The lack of a long-lived GSB recovery is evident (Supplementary Figure S2B,D) that per excitation pulse, the TA signal from any photoconverted population is likely within our experimental signal-to-noise ratio. Although having an apparently lower photoconversion efficiency than Apcb, Bbv still exhibit a fast photoconversion and can operate effectively under cw or ambient light sources for potential optogenetic applications $[3,7,17]$.

\subsection{Ground State Femtosecond Stimulated Raman Spectroscopy Sheds Light on Structural Factors} That Rationalize the Unique Reversible Photoswitching Pathways in Bbv

To help elucidate the atomic structures of Bpcb and Bbv conformers, GS-FSRS experiments were performed to obtain the vibrational signatures of $\mathrm{Apcb}, \mathrm{Bpcb}$, and Bbv (Figure 4). Respective LEDs were implemented to collect the Apcb $P_{r}$ and $P_{g}$ (Figure $4 A$ ), Bpcb $P_{r}$ and $\mathrm{P}_{\mathrm{g}}$ (Figure $4 \mathrm{~B}$ ), as well as Bbv $\mathrm{P}_{\mathrm{fr}}$ and $\mathrm{P}_{\mathrm{o}}$ conformers (Figure $4 \mathrm{C}$ ). With the pre-resonance ps $803 \mathrm{~nm}$ Raman pump, the fs broadband Raman probe on the Stokes side was used to effectively prevent notable dispersive line shapes of the cofactor chromophore [20,48]. The collected spectra in Figure 4A match our previous experimental results on Apcb using various Raman pump wavelengths at 792,678, and $596 \mathrm{~nm}$ with Raman probe on the Stokes or anti-Stokes sides [15]. As a control experiment, we also collected the anti-Stokes FSRS spectra with a bluer Raman probe than the Raman pump (Supplementary Figure S6) [22,48,49], which exhibit much stronger peaks due to the resonance enhancement achieved by the Raman probe with respect to the ground state absorption peaks of $P_{f r}(699 \mathrm{~nm})$ and $P_{0}$ $(624 \mathrm{~nm})$, further confirming the vibrational peak frequencies in $\mathrm{S}_{0}[28,48,50,51]$. Using the Gaussian software [52], density functional theory (DFT) calculations at B3LYP level with 6-31G(d,p) basis sets were performed on the BV cofactor to visualize the vibrational normal modes (see Experimental Methods in Section 3.4 below). A frequency scaling factor 0.97 was applied to match the DFT-calculated spectrum to the GS-FSRS spectrum of the Bbv $\mathrm{P}_{\mathrm{fr}}$ conformer (Supplementary Figure S7). The discrepancy in the Raman peak intensities is likely due to the DFT calculations only treating the cofactor quantum mechanically in vacuo without the protein pocket. More accurate hybrid quantum mechanics/molecular mechanics (QM/MM) methods would help model the CBCR protein with the embedded cofactor as a whole $[37,45,53,54]$ which could complement our experimental capabilities in 
ultrafast laser spectroscopy. The description of major vibrational modes of the $\mathrm{P}_{\mathrm{fr}}$ state can be seen in Supplementary Table S1.

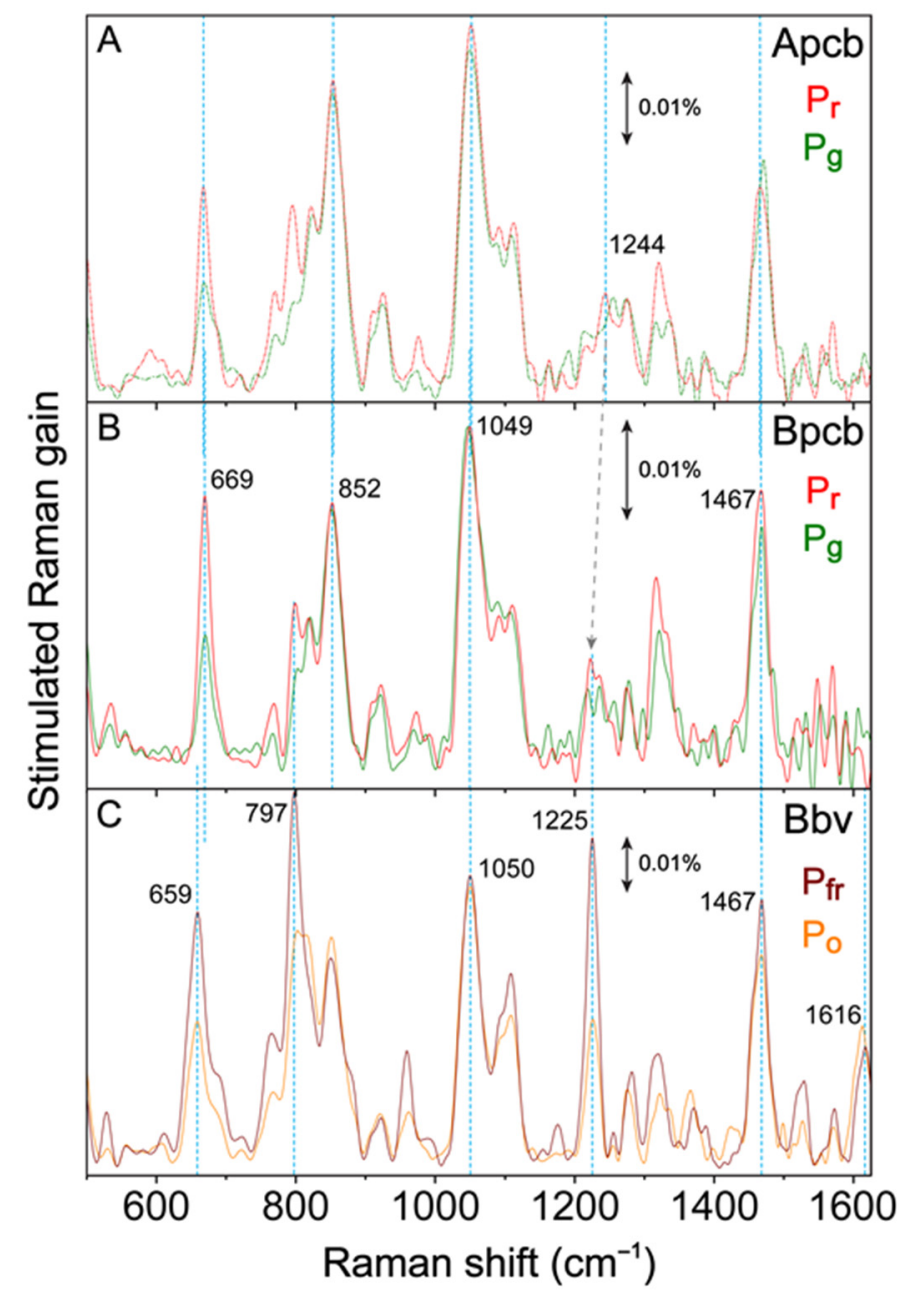

Figure 4. Raman signatures of various cofactors in related CBCR conformers. GS-FSRS spectra of (A) Apcb and (B) Bpcb $P_{\mathrm{r}}$ (red) and $\mathrm{P}_{\mathrm{g}}$ (green) species using 505 and $650 \mathrm{~nm}$ LEDs, respectively, as well as (C) Bbv $P_{\text {fr }}$ (dark red) and $P_{o}$ (orange) species using $600 \mathrm{~nm}$ LEDs and a $650 \mathrm{~nm}$ longpassfiltered tungsten lamp. The ps $803 \mathrm{~nm}$ Raman pump was used to minimize the pump-induced conversion of the cofactor species, with the fs Raman probe set at the Stokes side. Vertical dotted lines and a dashed arrow denote the peak frequency shifts between key vibrational modes of related CBCR cofactors. The double-headed arrows denote the stimulated Raman gain magnitude of $0.01 \%$ in each panel.

Overall, $\mathrm{Apcb}, \mathrm{Bpcb}$, and Bbv share marker bands common to bilin photosensors at $\sim 1051$ and $1466 \mathrm{~cm}^{-1}$ [35,55]. PCB and BV cofactors exhibit similar vibrational motions with small frequency shifts (Figure 4). The $1051 \mathrm{~cm}^{-1}$ mode is localized to the D-ring deformation with strong $\mathrm{C}-\mathrm{H}$ sidechain wagging. The $1466 \mathrm{~cm}^{-1}$ is attributed to the $\mathrm{B}$ and $\mathrm{C}$ ring-deformation and $\mathrm{CH}_{3}$ rocking. Both of these modes are stabilized by the conserved His322, Trp289, and Tyr352 in the CBCR pocket [18]. The overall higher intensity of the redabsorbing species $\left(\mathrm{P}_{\mathrm{r}}\right.$ in Apcb and Bpcb, and $\mathrm{P}_{\mathrm{fr}}$ in $\left.\mathrm{Bbv}\right)$ is attributed to better resonance enhancement with the $803 \mathrm{~nm}$ Raman pump. Moreover, the similar peak intensity at $\sim 1050 \mathrm{~cm}^{-1}$ for $P_{\mathrm{o}}$ and $\mathrm{P}_{\mathrm{fr}}$ of $\mathrm{Bbv}$ while most other modes display reduced intensities in $\mathrm{P}_{\mathrm{o}}$ can be rationalized by the vibronic coupling matrix that yields the mode-dependent change of resonance conditions $[15,20]$, which indicates the importance of light-induced D-ring motions that connect these conformers. Key differences between the vibrational 
spectra of PCB (Figure 4A,B) and BV (Figure 4C) can be seen by the red-shifted modes around 660 and $800 \mathrm{~cm}^{-1}$, and in the $1200-1400 \mathrm{~cm}^{-1}$ region.

The $\sim 660 \mathrm{~cm}^{-1}$ peak region reveals key differences in electronic conjugation between the PCB and BV cofactors. This Raman mode involves collective $\mathrm{C}-\mathrm{C}$ and $\mathrm{C}-\mathrm{H}$ wagging motions in the conjugated cofactor ring system. A frequency redshift of $10 \mathrm{~cm}^{-1}$ from the $\sim 669 \mathrm{~cm}^{-1}$ mode in Apcb and Bpcb (Figure 4A,B) to $659 \mathrm{~cm}^{-1}$ mode in Bbv (Figure $4 \mathrm{C}$ ) is rationalizable by two extra double bounds in the $\mathrm{A}$ and $\mathrm{D}$ rings of $\mathrm{Bbv}$ cofactor (see Figure 1C). In Bbv, the peak doublet around $825 \mathrm{~cm}^{-1}$ shows a clear blueshift of the lowerfrequency mode from $P_{\text {fr }}\left(797 \mathrm{~cm}^{-1}\right)$ to $P_{o}\left(809 \mathrm{~cm}^{-1}\right)$. Since the associated vibrational motions involve $\mathrm{N}-\mathrm{H}$ wagging from the $\mathrm{A}, \mathrm{B}$, and $\mathrm{C}$ rings with $\mathrm{C}-\mathrm{H}$ wagging on the $\mathrm{CD}$ bridge $[15,55]$, the frequency blueshift is likely due to a twisted $\mathrm{D}$ ring that disrupts the H-bonding of Trp289 to A ring. In Apcb and Bpcb, the lack of a strong peak around $800 \mathrm{~cm}^{-1}$ could be attributed to a different $\mathrm{H}$-bonding network of the A ring with reduced electric polarizability (one less double bond). In the $1200-1400 \mathrm{~cm}^{-1}$ region, Bbv exhibits a prominent $1225 \mathrm{~cm}^{-1}$ mode. In Bpcb and Apcb, this mode is weak but displays a noticeable redshift from Apcb to Bpcb ( 1244 to $1225 \mathrm{~cm}^{-1}$, see the tilted arrow from Figure $\left.4 \mathrm{~A}, \mathrm{~B}\right)$. In both PCB and BV calculations [15], since this mode involves delocalized $\mathrm{N}-\mathrm{H}$ rocking and $\mathrm{AB}$ bridge $\mathrm{C}-\mathrm{H}$ rocking motions, the clear peak enhancement in Bbv (Figure $4 \mathrm{C}$ ) further corroborates the shifted, stronger H-bonded A ring in the more conjugated BV cofactor. The redshift in Bpcb compared to Apcb is also consistent with the BV4 mutations that allow the PCB cofactor to adopt a slightly shifted position inside the protein pocket and establish better H-bonding interactions, yet the largely unchanged Raman peak intensity around $1235 \mathrm{~cm}^{-1}$ indicates that the mode-dependent electric polarizability is dictated by the intrinsic chromophore electronic structure (see the clear peak intensity ratio and line width change from Figure 4A,B to Figure 4C) [20,29].

\subsection{Contrasting Excited-State Potential Energy Surfaces for Reversible Photoswitching of PCB and BV Cofactors in the AnPixJg2_BV4 Pocket}

With all the correlated electronic and vibrational signatures from steady-state to timeresolved regime $[15,20]$, we could sketch the overall reversible photoswitching pathways of Bpcb and Bbv (Figure 5). Bpcb adopts the similar reaction scheme as Apcb as the BV4 mutations do not affect the major pathways of the embedded cofactor. The less bulky residues (particularly Thr308 and Val336, Figure 1B) in Bpcb allow the D ring more room to twist, decreasing the excited-state dynamics time constants $[15,36]$. In the Bpcb $\mathrm{P}_{\mathrm{g}} \rightarrow$ $\mathrm{P}_{\mathrm{r}}$ conversion (Figure 5A), the $\mathrm{P}_{\mathrm{g}}{ }^{*}$ species undergoes FC relaxation on the $\sim 100 \mathrm{fs}$ time scale, then approaches a CI on the $\sim 4.2$ ps time scale. The slightly shifted cofactor in Bpcb makes the initial twist slightly slower than that in Apcb ( 2.7 ps) [15]. After the CI, the cofactor population likely continues twisting to the converted Lumi-G state or twisting back to $\mathrm{P}_{\mathrm{g}}$ on the 20-30 ps time scales. This step is evident by a $\sim 20$ ps component in the Lumi-G rise (HGSA at $\sim 595 \mathrm{~nm}$ in Figure 2A) and a $\sim 30$ ps component in the GSB recovery (Supplementary Figure S2A). As for the opposite $P_{r} \rightarrow P_{g}$ conversion (Figure 5C), after FC relaxation the $\mathrm{P}_{\mathrm{r}}{ }^{*}$ species transitions into a twisted $\mathrm{P}_{\mathrm{r}}{ }^{* \prime}$ intermediate with a $4.5 \mathrm{ps}$ time constant, which requires further ring twists into a $\mathrm{CI}$ on the $111 \mathrm{ps}$ time scale. The $\mathrm{P}_{\mathrm{r}}{ }^{* \prime}$ state can be seen by the transient rise and decay of a weak ESA band around $615 \mathrm{~nm}$ (Figure 2C and Supplementary Figure S3), corroborated by a stronger ESA band around $616 \mathrm{~nm}$ with similar dynamics for the same PCB cofactor inside the AnPixJg2 pocket (i.e., Apcb) [15]. 

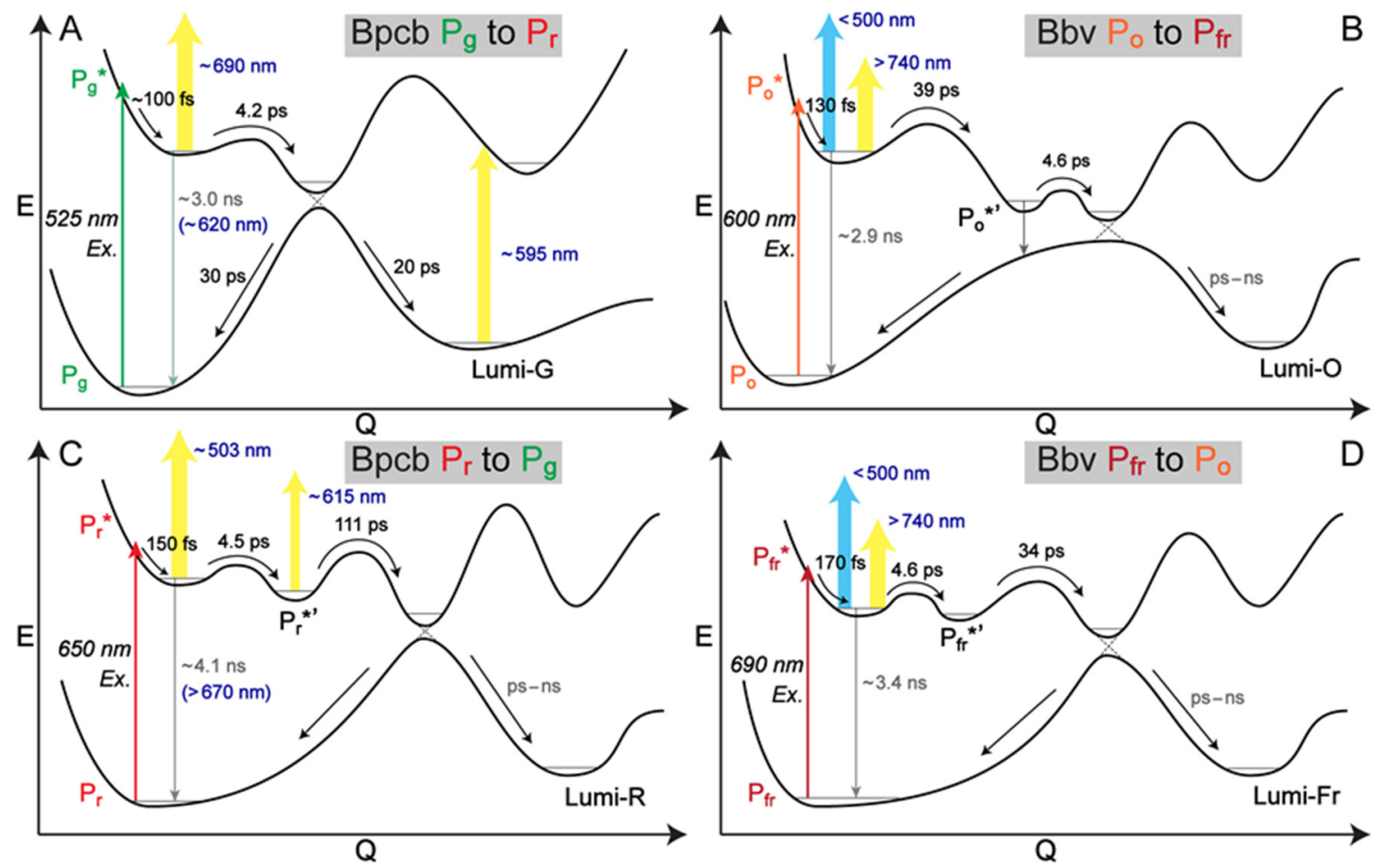

Figure 5. Representative potential energy surfaces of Bpcb (A) $P_{g} \rightarrow P_{r},(C) P_{r} \rightarrow P_{g}$, and Bbv (B) $P_{o} \rightarrow P_{f r},(D) P_{f r} \rightarrow P_{o}$ photoconversion. The photoexcitation (Ex.), ESA, SE, GSB, and HGSA transitions are illustrated by the color-coded vertical arrows. The arrow lengths are not drawn to scale with the exact energy gaps. Reaction scheme is denoted by curved arrows with characteristic time constants retrieved from global analysis of the corresponding TA spectra in Figures 2 and 3 . A minor fluorescence component is shown by a dim downward arrow. The ps-ns time scales for Lumi state formation in panels (B-D) indicate a long-lived state beyond our current detection time window. The horizontal axis " $Q$ " represents the dominant cofactor ring-twisting motions that could change as time progresses after photoexcitation, while the protein environment continuously contributes to the cofactor nuclear motions.

On the long time scale, since the 3-4 ns minor component from global analysis of $\mathrm{Bpcb}$ (Figure $3 \mathrm{~A}, \mathrm{~B}, \mathrm{E}, \mathrm{F}$ ) is an average value of all the long processes going beyond our current time window of $\sim 1 \mathrm{~ns}$, the pertinent contributors could be fluorescence from $\mathrm{P}_{\mathrm{g}}{ }^{*}$ species (see the $620 \mathrm{~nm}$ SE band in Figure 2A and PES in Figure 5A) or $\mathrm{P}_{\mathrm{r}}{ }^{*}$ species (see the $>670 \mathrm{~nm}$ SE band in Figure 2C and PES in Figure 5C), and the long-lasting photoproduct state that does not return to the original ground state on this time scale (e.g., Lumi-G in Figure 5A and Lumi-R in Figure 5C). In analogy, the similar $\sim 3$ ns minor component from global analysis of Bbv (Figure 3C,D,G,H) could involve weak fluorescence from $\mathrm{P}_{\mathrm{o}}{ }^{*}$ and $\mathrm{P}_{\mathrm{fr}}{ }^{*}$ species plus the long-lasting photoproduct states Lumi-O and Lumi-Fr in Figure $5 \mathrm{~B}$ and $5 \mathrm{D}$, respectively. It is interesting to note that these minor components with $1-4 \%$ amplitude weights were consistently retrieved from both the red ESA band $(\sim 740 \mathrm{~nm})$ and GSB band (633-639 nm) of Bbv (Supplementary Figure S2B,D), indicating that a small percentage of the excited-state population goes from the excited state to the original ground state on the $\sim 3$ ns time scale (a typical radiative transition lifetime), which could be due to the aforementioned weak fluorescence component. The absence of a prominent SE band could be due to spectral overlap with the strong and broad ESA band on the red side (Figure 2B,D and Figure 3C,G,D,H), and any mismatch between the ESA and GSB dynamics could imply some deviations from a direct $S_{1}$-to- $S_{0}$ transition. These long-time-scale processes could benefit from future in-depth studies with other experimental and computational techniques (see below) that are beyond the scope of this work.

Notably, the engineered Bbv undergoes a more "purely" reversible photoswitching pathway as reflected by the highly conserved excited-state time constants from either conformer of the cofactor. The $\mathrm{P}_{\mathrm{fr}} \rightarrow \mathrm{P}_{\mathrm{o}}$ conversion (Figure 5D) adopts a similar scheme to 
the $\mathrm{P}_{\mathrm{r}} \rightarrow \mathrm{P}_{\mathrm{g}}$ conversion of the PCB samples from the thermally equilibrated state to the metastable state. The similarly weighted $\sim 4.6$ and 34 ps time constants infer that the $4.6 \mathrm{ps}$ small-scale twisting motion occurs prior to the 34 ps larger twist, which is characteristic of the D-dihedral change acting as a preparatory step [28,56] for the C-dihedral change (see details in Section 2.3 above). In contrast, starting from the metastable state with a more downhill relaxation, the higher-weighted $\sim 39$ ps larger twisting motion could generate a $P_{0}{ }^{* \prime}$ species that is energetically closer to the ground state, which allows for other nonradiative processes (depicted by a vertically downward gray arrow in Figure 5B) to more effectively compete with the 4.6 ps small-scale twist leading into to a CI region before the Lumi-O formation. Given this kinetic scheme, we do not expect a significant accumulation of transient $P_{\mathrm{o}}{ }^{* \prime}$ species since the 4.6 ps outgoing rate is much faster than the $39 \mathrm{ps}$ incoming rate, which likely explains the lack of clear TA features from the $\mathrm{P}_{\mathrm{o}}{ }^{*}$ species. As a result, the observed TA dynamics (Figure 2B) mainly reflect the ultrafast multiexponential decay of $\mathrm{P}_{\mathrm{o}}{ }^{*}$ species (Figure $5 \mathrm{~B}$ ), consistent with kinetic models with reversible reactions connecting all the transient species. In particular, the DADS feature with a 4.6 ps lifetime (Figure 3G) does not reflect the $P_{\mathrm{o}}{ }^{* \prime}$ species per se, instead, it mathematically tracks the 4.6 ps kinetic component (along the $\mathrm{P}_{\mathrm{o}}{ }^{*}$-to-CI pathway) with a similar spectral profile as the initial $\sim 130 \mathrm{fs}$ and subsequent 39 ps components (i.e., $\mathrm{P}_{\mathrm{O}}{ }^{*}$ species) as shown in the EADS analysis (Figure 3C).

Recent reports on the ultrafast dynamics of a red/far-red bathy bacteriophytochrome named PaBphP that binds BV inside the GAF domain (part of a classical PAS-GAF-PHY architecture) [32,36] provide a useful comparison. The forward ${ }^{15 Z} \mathrm{P}_{\mathrm{r}} \rightarrow{ }^{15 E} \mathrm{P}_{\mathrm{fr}}$ photoconversion utilizes a two-step process on the excited state with a 2-207 ps local environment (e.g., protein residue sidechains, internal/trapped water molecules [55-57], elaborate H-bonding networks) relaxation and a 375 ps isomerization to the $\mathrm{CI}$ [32]. For comparison, the reverse $\mathrm{P}_{\mathrm{fr}} \rightarrow \mathrm{P}_{\mathrm{r}}$ photoconversion (with higher bond strain/distortion to release) goes through an excited-state bifurcation on the much faster $\sim 1$ and 4 ps time scales where both wavepackets reach the $\mathrm{CI}[36]$. The pertinent few-ps photoisomerization step around the $\mathrm{C} 15=\mathrm{C} 16$ bond that significantly shifts the cofactor D-ring with respect to a nearby protein pocket residue is consistent with our assignment of the 3-5 ps component to the D-dihedral/small-scale twisting motion (see Section 2.3 above). By evaluating the conformational differences between $\mathrm{P}_{\text {fr }}$ and $\mathrm{P}_{\mathrm{r}}$ states of $\mathrm{PaBphP}$ via X-ray crystallography, the $\mathrm{BV}$ cofactor exhibits distinct conformations of the $\mathrm{A}, \mathrm{B}, \mathrm{C}$, and $\mathrm{D}$ rings [58], which underlie the significant difference between forward and reverse pathways mediated by different residues in the extensively rearranged $\mathrm{H}$-bonding network. In sharp contrast, stabilizing the $\mathrm{A}, \mathrm{B}$, and $\mathrm{C}$ rings through largely conserved H-bonding and $\pi-\pi$ stacking (e.g., between Trp289 and D ring) in the BV4 pocket [18] could therefore allow a rather unique and "purely" reversible photoconversion pathway of the $\mathrm{BV}$ cofactor (between the $\mathrm{P}_{\mathrm{o}}$ and $\mathrm{P}_{\mathrm{fr}}$ states of $\mathrm{Bbv}$, Figure 1F) as observed in this work (Figures 2 and 3, Supplementary Figures S1 and S2). Notably, the focus here is the elucidation of reversible photoswitching between distinct (not necessarily pure) conformers in the protein pocket.

The more homogeneous, thermally equilibrated populations of Bpcb and Bbv could also be related to the protonation state of the adjacent His322 [37]. QM/MM calculations indicate that the His322 protonation state is a trigger for structural heterogeneity. In the crystal structure of the $\mathrm{P}_{\text {fr }}$ state of AnPixJg2_BV4 [18], His322 shares two H-bonding partners: one is the $\mathrm{B}$ ring propionate, the other is a local water molecule that also $\mathrm{H}$-bonds to the D ring nitrogen site (-NH group). The His318Tyr mutation in BV4 removes the original H-bond between His318 and the B ring propionate in AnPixJg2 [8] and would thus increase the H-bonding interaction between $\mathrm{B}$ ring and His322 [18]. For the other $\mathrm{H}$-bonding partner, since both $\mathrm{PCB}$ and $\mathrm{BV}$ cofactors undergo some position shift in the $\mathrm{BV} 4$ pocket, the $\mathrm{D}$ ring likely has more tendency to move away from His322. The resultant singly protonated state of His322 (as an H-bond donor to the B ring propionate) would support a more homogeneous cofactor population [37], which is evident from the "native" steady-state electronic absorption spectra that show Bbv (Figure 1F, black trace) being 
more homogeneous than Bpcb (Figure 1E, black trace). The homogeneous population also supports Bbv in adopting a sequential kinetic model instead of a parallel model to undergo excited-state electronic and structural relaxation of the BV cofactor with continuous activesite motions in $\mathrm{S}_{1}[30,32]$, followed by slower thermal relaxation events of the chromophore and protein matrix in the electronic ground state $\left(\mathrm{S}_{0}\right)$. By exciting a mostly homogeneous ground state population, it is unlikely to have the transient wavepacket branch into different pathways with notably different time constants (e.g., $\sim 5$ and $35 \mathrm{ps}$ ) that both reach the CI.

Meanwhile, the $\mathrm{P}_{\mathrm{o}}$ conformer of Bbv is likely more homogeneous due to its higher absorption peak than the $\mathrm{P}_{\mathrm{g}}$ conformers of Apcb and Bpcb (Figure 1F orange trace vs. Figure 1D,E green traces, all relative to their corresponding redder-absorbing peaks), because a larger extinction coefficient could be indicative of a more stabilized $\mathrm{P}_{\mathrm{o}}$ state of Bbv. For corroboration, implementing the BV4 mutation to AnPixJg4 (the fourth GAF domain of AnPixJ) leads to a rapid $\mathrm{P}_{\mathrm{o}}$-to- $\mathrm{P}_{\mathrm{fr}}$ dark reversion that is $\sim 400$-fold faster than that of AnPixJg2_BV4 (Bbv), while the relative $\mathrm{P}_{\mathrm{o}} / \mathrm{P}_{\text {fr }}$ absorption peak intensity ratio $(0.65$, peak wavelengths at $628 \mathrm{~nm} / 700 \mathrm{~nm}$ ) [18] is indeed smaller in AnPixJg4_BV4 than that in $\operatorname{Bbv}(0.85$, peak wavelengths at $624 \mathrm{~nm} / 699 \mathrm{~nm}$, Figure 1F). The BV-binding efficiency of AnPixJg4_BV4 (44\%) is also significantly lower than AnPixJg2_BV4 (75\%). Further investigation of AnPixJg4_BV4 using a similar combination of fs-TA and GS-FSRS to this work could help to unveil the mechanistic origin for the fourth GAF domain to exhibit greatly accelerated dark reversion on the ground state, shedding light on the effect of cofactor-protein-pocket interplay on the apparent TA features versus the thermally equilibrated $\mathrm{P}_{\mathrm{fr}}$ state. Knowledge about the effects of specific attributes around the cofactor and how they contribute to the overall photoswitching dynamics would enable the protein engineers to effectively tailor these photoswitching proteins for specific applications.

To further decipher the multidimensional $[20,56,57]$ photoswitching dynamics, we expect the improved molecular dynamics (MD) simulation methods with higher accuracy and longer duration $[26,37,55,57,59]$ and other spectroscopic techniques to paint a holistic and accurate portrait as the molecular system is excited, photoconverting, and relaxing back to its thermally stable dark-adapted state $[29,55]$. Since the metastable light-adapted $\mathrm{P}_{\mathrm{o}}$ and $\mathrm{P}_{\mathrm{g}}$ crystal structures are challenging to obtain, $\mathrm{QM} / \mathrm{MM}$ methodology has advanced in closely matching the calculated absorption spectra of protein conformers with their experimental counterparts, including recent successes on a similar red/green absorbing CBCR named Slr1393g3 with its $P_{r}$ and $P_{g}$ crystal structures both solved $[38,54]$. By implementing similar $\mathrm{QM} / \mathrm{MM}$ calculations on Bbv, more accurate vibrational and structural insights into the $\mathrm{P}_{\mathrm{O}}$ conformer could be gained to augment our current understanding of the $\mathrm{P}_{\mathrm{fr}}$ conformer (Figure 4 and Table S1) with its available crystal structure [18]. Moreover, a systematic calculation of the excited-state free energies of the BV cofactor with various D-ring twisting geometries and intermediates (see Section 2.3 above) in the protein pocket can shed more light on the ultrafast reversible photoswitching pathway between the $P_{o}$ and $\mathrm{P}_{\mathrm{fr}}$ conformers of AnPixJg2_BV4.

In addition, an expanded spectroscopic toolset from ultrafast regime (typically fs-to-ns time scales as reported here) to the more macroscopic time scales (ns-to-ms or longer) could better identify long-lived or later intermediates (e.g., Lumi-G and Lumi-R in Apcb and Bpcb, and Lumi-O and Lumi-Fr in Bbv), which may dictate the forward/reverse reaction speed and yield $[5,29,60,61]$. These Lumi states are likely similar to the photoproduct species in our current experiments on the ns time scale (Figure 5), however, they require further protein relaxation and chromophore pocket rearrangement on the electronic ground state to eventually reach the final product state. For example, a TA setup with pulsed (100 ns) LED-based broadband probe has expanded the time window from $\mathrm{ns}$ to $\mathrm{ms}$ and identified two intermediates between Lumi- $G_{2}$ and $P_{r}$ after $532 \mathrm{~nm}$ excitation of $P_{g}$ of NpR6012g4 [62], and recent applications in conjunction with fs-TA were able to elucidate a series of meta states that emerge after the Lumi state formation in AnPixJg2 as well as NpR6012g4 with a strong sequence homology (both are canonical red/green CBCRs) [61]. In aggregate, by combining multiple techniques such as TA, FSRS, QM/MM, X-ray crystallography, and 
NMR, the resultant comprehensive information can delineate the complex photoconversion of CBCRs and other photosensing proteins as they convert from one state to another on various time scales. Such a more complete understanding of the working mechanisms of these fascinating nanomachines can help more bioengineers to better predict, design, prepare, and tune the next generation of bioimaging and optogenetic toolsets to advance life sciences and improve human health.

\section{Materials and Methods}

\subsection{Protein Expression}

The His-tagged AnPixJg2_BV4 inserted into pET28a vector (Novagen, Sigma Aldrich, St. Louis, MO, USA) was constructed in our previous study [18]. The plasmid was transferred into the E. coli strain C41 (Cosmo Bio, Tokyo, Japan) harboring the PCB synthetic system (pKT271-C0185) or BV synthetic system (pKT270) for protein expression. The bacterial cells were grown on Lysogeny Broth (LB) agar medium at $37^{\circ} \mathrm{C}$ and selected by kanamycin and chloramphenicol (each final concentration set at $20 \mu \mathrm{g} / \mathrm{mL}$ ), then cultured in $1 \mathrm{~L} \mathrm{LB}$ medium at $37^{\circ} \mathrm{C}$ until the cell optical density (OD) at $600 \mathrm{~nm}$ reached a range of $0.4-0.8$. Isopropyl $\beta$-D-1-thiogalactopyranoside (IPTG at final concentration of $0.1 \mathrm{mM}$ ) was then added into the culture media and these cells were cultured at $18{ }^{\circ} \mathrm{C}$ overnight to induce protein expression.

\subsection{Protein Extraction and Purification}

After protein expression was induced, the culture broth was centrifuged at $5000 \mathrm{~g}$ for $15 \mathrm{~min}$ to collect cells and then frozen at $-80^{\circ} \mathrm{C}$. The cells were suspended in a lysis buffer (20 mM HEPES-NaOH at $\mathrm{pH}=7.5,0.1 \mathrm{M} \mathrm{NaCl}$, and $10 \%(w / v)$ glycerol) and disrupted by Emulsiflex C5 high-pressure homogenizer at 12,000 psi (Avestin Inc., Ottawa, On, Canada), The 4-(2-hydroxyethyl)piperazine-(2-ethanesulfonic acid) or HEPES aqueous buffer solution was used because it has higher stability in maintaining the physiological $\mathrm{pH}$ values of cell culture media despite changes in $\mathrm{CO}_{2}$ concentration. The mixtures were then centrifuged at $165,000 \times g$ for $30 \mathrm{~min}$ to remove pellets, and the collected solutions were filtered through a $0.2 \mu \mathrm{m}$ membrane. After adding imidazole ( $30 \mathrm{mM}$ final concentration), the solution was loaded onto a nickel-affinity His-trap column (GE Healthcare, Chicago, IL, USA). After washing with lysis buffer containing 30-100 mM imidazole, the His-tagged proteins were purified using lysis buffer containing $100-400 \mathrm{mM}$ imidazole with a linear gradient system $(1 \mathrm{~mL} / \mathrm{min})$ for $15 \mathrm{~min}$. The purified proteins were incubated with $1 \mathrm{mM}$ EDTA on ice for $1 \mathrm{~h}$ and then dialyzed against the lysis buffer to remove the residual imidazole and EDTA. This detailed process to make Bpcb (green/red) and Bbv (orange/far-red) samples in this work was identical to the one that we used to prepare Apcb (green/red) samples [15], and all the CBCR samples were prepared in the aforementioned HEPES buffer solution at physiological $\mathrm{pH}$ (7.5) and the specific concentrations (see Table 1 in Section 2.1 above) for all the steady-state and time-resolved spectroscopic measurements as reported herein.

\subsection{Femtosecond Transient Absorption ( $f_{S}-T A$ ) and Ground-State Femtosecond Stimulated Raman Spectroscopy (GS-FSRS) with Various Light Irradiation Conditions}

Our fs-TA and GS-FSRS setups have been built upon a Ti:sapphire-based laser oscillator (Mantis-5, Coherent, Inc., Santa Clara, CA, USA) and regenerative amplifier (Legend Elite-USP-1K-HE, Coherent, Inc.), which produce fundamental pulses of $\sim 800 \mathrm{~nm}$ center wavelength, $3.7 \mathrm{~W}$ average power, and $35 \mathrm{fs}$ duration at $1 \mathrm{kHz}$ repetition rate $[57,63]$. Tunable fs photoexcitation pulses were generated through a home-built two-stage noncollinear optical parametric amplifier (NOPA) [64]. The desired output laser wavelength was selected in the first NOPA by overlapping a supercontinuum white light (SCWL) and a $400 \mathrm{~nm}$ pump, which were generated by focusing a portion of the fundamental pulses on a thin sapphire crystal and a BBO crystal, respectively. This output pulse was used as the seed and further amplified by the second NOPA with a stronger $400 \mathrm{~nm}$ pump. The 
probe pulse was also an SCWL but generated through a 2-mm-thick quartz cell filled with deionized water to extend to a bluer spectral region than the sapphire plate [31]. The actinic pump and probe pulses were temporally compressed via chirped-mirror pairs DCM-12 (400-700 nm) and DCM-9 (450-950 nm, Laser Quantum, Inc., Stockport, UK), respectively [15]. The laser beam size at the focus for the actinic pump was measured to be $\sim 150 \mu \mathrm{m}$ in diameter. The pump-probe delay was controlled by a $150 \mathrm{~mm}$ motorized linear translation stage (NRT150, Thorlabs Inc., Newton, NJ, USA) that enables a detection time window of $\sim 1 \mathrm{~ns}$. To ensure sufficient data points across the entire time window for subsequent spectral analysis up to the ns time scale (see Figure 3 and Supplementary Figure S2), we collected a total of 110 time delay points between -2 ps and 600 ps (taking every $100 \mathrm{ps}$ from 100 to $600 \mathrm{ps}$, hence 6 points on the long time scale) for the $\mathrm{Bpcb} \mathrm{P}_{\mathrm{g}} \rightarrow$ $\mathrm{P}_{\mathrm{r}}$ transition (Figure 2A), and a total of 138 time delay points between $-2 \mathrm{ps}$ and $900 \mathrm{ps}$ (every 50 ps from 100 to 900 ps, hence 17 points on the long time scale) for the Bpcb $P_{r}$ $\rightarrow \mathrm{P}_{\mathrm{g}}, \mathrm{Bbv} \mathrm{P}_{\mathrm{o}} \rightarrow \mathrm{P}_{\mathrm{fr}}$, and Bbv $\mathrm{P}_{\mathrm{fr}} \rightarrow \mathrm{P}_{\mathrm{o}}$ transitions (Figure 2C,B,D). In particular, for the probe-dependent fits of TA marker bands for Bbv (Supplementary Figure S2B,D), we fit the long time constant within a numerical range from $\sim 1-5 \mathrm{~ns}$, and found that the best fits to go through 17 data points from time delay of 100 to 900 ps without large offsets were achieved with a $\sim 3$ ns time constant. These characteristic values were corroborated by global analysis results (see Figure 3C,G,D,H), which show a $~ 10 \%$ increase of root-mean-square deviation (RMSD) if the ns time constant was removed from the fits. This systematic retrieval of time constants and amplitudes in Supplementary Figure S2 in association with fs-TA data in Figure 2 substantiates all our reaction models in Figure 5, further supported by our FSRS data in Figure 4 (see Section 2).

Before each fs-TA experiment, the CBCR sample solution inside a $2 \mathrm{~mL}$ microcentrifuge tube was kept in a home-built 3D-printed black box and irradiated with LEDs of a certain wavelength for $5 \mathrm{~min}$ to ensure that all protein samples were converted to the desired state. The detail of the 3D LED box can be found in our previous report [15]. The sample solution was then circulated through a 1-mm-pathlength quartz flow cell (48-Q-1, Starna Cells, Inc., Atascadero, CA, USA) positioned in the laser beam path. During the experiments, the power of actinic pump that can convert the sample was set at $\sim 0.3 \mathrm{~mW}$ while the LEDs were constantly on to ensure the sample return to their initial states after the laser-induced conversion. Detailed combinations of the pump and LED wavelengths are listed in Table 1 (see above) as a handy summary for experimental conditions that yield major results in this work.

For GS-FSRS experiments (note that "femtosecond" in the FSRS terminology does not restrict it to excited-state measurements using a preceding actinic pump, so only the Raman ps-pump-fs-probe pair is required for a ground-state measurement), the $\sim 4 \mathrm{~mW}$, 2 ps Raman pump with $803 \mathrm{~nm}$ center wavelength was directly produced from a portion of the laser fundamental output through a home-built reflective-grating-slit-based spectral filter [63]. This pump wavelength was shown to lead to minimal photoconversion that could generate a mixed $\mathrm{P}_{\mathrm{g}} / \mathrm{P}_{\mathrm{r}}$ population of AnPixJg2 [15] so we can focus on the more pure conformer state under specific cw light irradiation conditions (see Figure 4). Raman pump was chopped at half of the laser repetition rate $(500 \mathrm{~Hz})$. The Raman probe was generated via a 2-mm-thick sapphire crystal plate (instead of a water-filled cuvette) due to its capability of producing stronger redder photons up to $\sim 920 \mathrm{~nm}[31,63]$. The same quartz flow cell and LED boxes were used. Both the Stokes and anti-Stokes GS-FSRS spectra were collected for a direct comparison and confirmation of Raman peak frequencies [20,49]. Since both fs-TA and GS-FSRS signals were generated along the probe direction, the probe beam passing through the sample solution was collimated and focused into a spectrograph (IsoPlane SCT-320, Princeton Instruments, Inc., Trenton, NJ, USA) with the reflective grating selected for TA (300 grooves/mm, $300 \mathrm{~nm}$ blaze wavelength) or FSRS (600 grooves/mm, $1 \mu \mathrm{m}$ blaze wavelength), then imaged at the exit focal plane onto a front-illuminated CCD array camera (PIXIS:100F, Princeton Instruments, Inc., Trenton, NJ, USA) with data output to a computer and further processing and storage by a custom LabVIEW suite. The 
obtained GS-FSRS data can be directly compared with the DFT calculation results of the cofactor molecule (see below) $[15,20]$.

\subsection{Quantum Calculations}

Ground state vibrational normal modes of the BV cofactor of AnPixJg2_BV4 mutant were calculated using Gaussian 16 software [52] at the DFT RB3LYP level of theory in vacuo. To generate the initial input of $\mathrm{BV}$ in the $\mathrm{P}_{\mathrm{fr}}$ form for calculation, the chromophore structure was first taken from the reported crystal structure (PDB ID: 5ZOH) [18], then proper numbers of hydrogen atoms were added and the two propionate groups on the $\mathrm{B}$ and $C$ rings were cut and capped with methyl groups $[15,45]$ in GaussView6. The structure was optimized with a series of basis sets from 3-21G, 6-31G to 6-31G(d,p), followed by vibrational frequency calculation of the geometrically optimized structure.

To investigate the effect of structural restraints of the chromophore on the calculation results, we examined a few different combinations of fixing the dihedral angles (e.g., CCCC and $\mathrm{CCCN}$ ) and bridge angles (i.e., the CCC angle along the methine bridge) between the chromophore rings: no angles were fixed between the four rings; two dihedral angles between $C / D$ rings and the bridge angle between $B / C$ rings were fixed; four dihedral angles between $A / B$, and $C / D$ rings as well as the bridge angle between $B / C$ rings were fixed; and all six dihedral angles between $A / B / C / D$ rings were fixed. The outcome of fixing all six dihedral angles of the cofactor matches the experimental results the best. Due to no crystal structure of $\mathrm{BV}$ in the $\mathrm{P}_{\mathrm{o}}$ form, the dihedral angle closer to the $\mathrm{D}$ ring (around the C15=C16 bond, see Figure 1C) in the ${ }^{15 Z} \mathrm{P}_{\text {fr }}$ form from crystal structure (PDB ID: $5 Z \mathrm{ZH}$ ) [18] was twisted to $180^{\circ}$ (to represent the ${ }^{15 E} \mathrm{P}_{\mathrm{o}}$ conformer, also see Graphical Abstract) and all six dihedral angles between the four rings were then fixed. The same calculation steps as those for the $P_{\text {fr }}$ form were followed. Due to the lack of accurate modeling of the pertinent interactions or related crystal structures with the $P_{o}$ conformer (especially for the translocated cofactor with a still-twisted A ring versus the $P_{f r}$ conformer [18]), there is a poor match between the calculated and observed Raman spectrum of the $P_{o}$ state, hence we focus on the more reliable mode assignment of the $P_{\text {fr }}$ state (see Supplementary Figure S7 and Supplementary Table S1). The Raman spectrum was exported with $7 \mathrm{~cm}^{-1}$ half widths at half height (HWHH) and $1 \mathrm{~cm}^{-1}$ step size. A scaling factor of 0.97 was used $[15,65]$ for the low and high-frequency regions to better match the experimental FSRS spectra (see Figure 4).

\section{Conclusions}

In summary, we systematically investigated Apcb, Bpcb, and Bbv as related CBCRs in aqueous buffer solution using the steady-state and time-resolved (fs-to-ns time scales) spectroscopic techniques, and revealed that the strategic BV4 mutations allow more room for the protein cofactor to shift and twist, achieve a more homogeneous native population of the embedded cofactor, and conserve the reversible photoconversion pathways as primary forward and reverse reaction events. When BV is incorporated into the AnPixJg2_BV4 pocket, the extended electronic conjugation along with the BV translocation essentially stabilize the A ring through an improved H-bonding network at the active site. The resulting conformational stability allows the ultrafast photoswitching dynamics to be more localized at the cofactor D ring, which undergoes characteristic small and largescale D-ring twisting motions with $\sim 5$ and 35 ps time constants upon photoexcitation. These primary events effectively lead to a clockwise/counterclockwise reversible pathway (en route to the $S_{1}-S_{0} C I$, followed by further relaxation in $S_{0}$ ) between the ${ }^{15 Z} \mathrm{P}_{\text {fr }}$ and ${ }^{15 E} \mathrm{P}_{\mathrm{o}}$ conformers. With the red-shifted absorption and emission properties, the targeted and effective BV4 mutation thus enables CBCRs to be visualized and regulated in deep mammalian tissues. The aforementioned more directional and controllable reversible photoconversion as the "central dogma" of phytochromes [2] for a homogeneous cofactor population (in the photosensory unit) could provide more collective and strong driving forces for the downstream signaling and regulatory events (e.g., transcription, motility). 
Given the vast $\mathrm{CBCR}$ family with various functions and properties, the ultrafast electronic and vibrational spectroscopic toolsets with complementary long-time-delay spectroscopic techniques, as well as MD and QM/MM calculations will continue to represent a powerful characterization platform in delineating the photoconversion mechanisms on molecular time scales and, more generally, unlocking the design space and potential from the bottom up for light-driven molecular machines for diverse applications.

Supplementary Materials: The following are available online at https:/ /www.mdpi.com/article/10 .3390/ijms22105252/s1.

Author Contributions: Conceptualization, C.F., M.S. and R.N.; methodology, S.R.T., L.T., L.Z., Y.T., K.F., Y.U., T.N. and Y.K.; software, S.R.T. and L.T.; formal analysis, S.R.T. and L.T.; investigation, S.R.T., L.T., Y.T. and K.F.; visualization, S.R.T. and C.F.; writing—original draft, S.R.T. and L.T.; writing - review and editing, C.F., M.S. and R.N.; supervision, C.F.; funding acquisition, C.F., M.S. and R.N. All authors have read and agreed to the published version of the manuscript.

Funding: This research was funded by the U.S. NSF grants (MCB-1817949 and CHE-2003550) to C.F. as well as the Japanese Science and Technology Agency, Core Research for Evolutional Science and Technology (JPMJCR1653 to M.S. and R.N.). We thank additional lab personnel support provided by the U.S. NSF MRI grant (DMR-1920368).

Institutional Review Board Statement: Not applicable.

Informed Consent Statement: Not applicable.

Data Availability Statement: The data presented in this study are available from the corresponding author upon request.

Acknowledgments: We appreciate the U.S. NSF East Asia and Pacific Summer Institutes for U.S. Graduate Students (EAPSI) and Japan Society for the Promotion of Science (JSPS) for Summer 2017 research fellowship support to S.R.T. We thank Cheng Chen and Taylor Krueger for helpful discussions.

Conflicts of Interest: The authors declare no conflict of interest.

$\begin{array}{ll}\text { Abbreviations } \\ \text { CBCR } & \text { cyanobacteriochrome } \\ \text { PCB } & \text { phycocyanobilin } \\ \text { BV } & \text { biliverdin } \\ \text { fs-TA } & \text { femtosecond transient absorption } \\ \text { GS-FSRS } & \text { ground-state femtosecond stimulated Raman spectroscopy } \\ \text { LED } & \text { light-emitting diode } \\ \text { CI } & \text { conical intersection } \\ \text { Apcb } & \text { AnPixJg2 with PCB cofactor } \\ \text { Bpcb } & \text { AnPixJg2_BV4 with PCB cofactor } \\ \text { Bbv } & \text { AnPixJg2_BV4 with BV cofactor } \\ \text { PDB } & \text { protein data bank } \\ \text { DFT } & \text { density functional theory } \\ \text { PES } & \text { potential energy surface } \\ \text { ESA } & \text { excited-state absorption } \\ \text { HGSA } & \text { hot ground-state absorption } \\ \text { GSB } & \text { ground-state bleaching } \\ \text { SE } & \text { stimulated emission } \\ \text { EADS } & \text { evolution-associated difference spectrum } \\ \text { DADS } & \text { decay-associated difference spectrum } \\ \text { FC } & \text { Franck-Condon } \\ \text { QM/MM } & \text { quantum mechanics/molecular mechanics } \\ \text { MD } & \text { molecular dynamics } \\ & \end{array}$




\section{References}

1. $\mathrm{Wu}, \mathrm{S} .-\mathrm{H} . ;$ Lagarias, J.C. Defining the bilin lyase domain: Lessons from the extended phytochrome superfamily. Biochemistry 2000, 39, 13487-13495. [CrossRef] [PubMed]

2. Rockwell, N.C.; Su, Y.-S.; Lagarias, J.C. Phytochrome structure and signaling mechanisms. Annu. Rev. Plant Biol. 2006, 57, 837-858. [CrossRef]

3. Ikeuchi, M.; Ishizuka, T. Cyanobacteriochromes: A new superfamily of tetrapyrrole-binding photoreceptors in cyanobacteria. Photochem. Photobiol. Sci. 2008, 7, 1159-1167. [CrossRef] [PubMed]

4. Hirose, Y.; Shimada, T.; Narikawa, R.; Katayama, M.; Ikeuchi, M. Cyanobacteriochrome CcaS is the green light receptor that induces the expression of phycobilisome linker protein. Proc. Natl. Acad. Sci. USA 2008, 105, 9528-9533. [CrossRef]

5. Narikawa, R.; Fukushima, Y.; Ishizuka, T.; Itoh, S.; Ikeuchi, M. A novel photoactive GAF domain of cyanobacteriochrome AnPixJ that shows reversible green/red photoconversion. J. Mol. Biol. 2008, 380, 844-855. [CrossRef] [PubMed]

6. Rockwell, N.C.; Lagarias, J.C. A brief history of phytochromes. ChemPhysChem 2010, 11, 1172-1180. [CrossRef]

7. Rockwell, N.C.; Martin, S.S.; Lagarias, J.C. Red/green cyanobacteriochromes: Sensors of color and power. Biochemistry 2012, 51, 9667-9677. [CrossRef]

8. Narikawa, R.; Ishizuka, T.; Muraki, N.; Shiba, T.; Kurisu, G.; Ikeuchi, M. Structures of cyanobacteriochromes from phototaxis regulators AnPixJ and TePixJ reveal general and specific photoconversion mechanism. Proc. Natl. Acad. Sci. USA 2013, 110, 918-923. [CrossRef]

9. Piatkevich, K.D.; Subach, F.V.; Verkhusha, V.V. Engineering of bacterial phytochromes for near-infrared imaging, sensing, and light-control in mammals. Chem. Soc. Rev. 2013, 42, 3441-3452. [CrossRef]

10. Ryu, M.-H.; Gomelsky, M. Near-infrared light responsive synthetic c-di-GMP module for optogenetic applications. ACS Synth. Biol. 2014, 3, 802-810. [CrossRef]

11. Ziegler, T.; Möglich, A. Photoreceptor engineering. Front. Mol. Biosci. 2015, 2, 30. [CrossRef] [PubMed]

12. Shu, X.; Royant, A.; Lin, M.Z.; Aguilera, T.A.; Lev-Ram, V.; Steinbach, P.A.; Tsien, R.Y. Mammalian expression of infrared fluorescent proteins engineered from a bacterial phytochrome. Science 2009, 324, 804-807. [CrossRef]

13. Filonov, G.S.; Piatkevich, K.D.; Ting, L.-M.; Zhang, J.; Kim, K.; Verkhusha, V.V. Bright and stable near-infrared fluorescent protein for in vivo imaging. Nat. Biotechnol. 2011, 29, 757-761. [CrossRef] [PubMed]

14. Narikawa, R.; Muraki, N.; Shiba, T.; Ikeuchi, M.; Kurisu, G. Crystallization and preliminary X-ray studies of the chromophorebinding domain of cyanobacteriochrome AnPixJ from Anabaena sp. PCC 7120. Acta Cryst. Sect. F 2009, F65, 159-162. [CrossRef]

15. Tachibana, S.R.; Tang, L.; Chen, C.; Zhu, L.; Takeda, Y.; Fushimi, K.; Seevers, T.K.; Narikawa, R.; Sato, M.; Fang, C. Transient electronic and vibrational signatures during reversible photoswitching of a cyanobacteriochrome photoreceptor. Spectrochim. Acta A 2021, 250, 119379. [CrossRef] [PubMed]

16. Auldridge, M.E.; Forest, K.T. Bacterial phytochromes: More than meets the light. Crit. Rev. Biochem. Mol. Biol. 2011, 46, 67-88. [CrossRef] [PubMed]

17. Oliinyk, O.S.; Chernov, K.G.; Verkhusha, V.V. Bacterial phytochromes, cyanobacteriochromes and allophycocyanins as a source of near-infrared fluorescent probes. Int. J. Mol. Sci. 2017, 18, 1691. [CrossRef] [PubMed]

18. Fushimi, K.; Miyazaki, T.; Kuwasaki, Y.; Nakajima, T.; Yamamoto, T.; Suzuki, K.; Ueda, Y.; Miyake, K.; Takeda, Y.; Choi, J.-H.; et al. Rational conversion of chromophore selectivity of cyanobacteriochromes to accept mammalian intrinsic biliverdin. Proc. Natl. Acad. Sci. USA 2019, 116, 8301-8309. [CrossRef] [PubMed]

19. Kennis, J.T.M.; Groot, M.-L. Ultrafast spectroscopy of biological photoreceptors. Curr. Opin. Struct. Biol. 2007, 17, 623-630. [CrossRef]

20. Fang, C.; Tang, L.; Chen, C. Unveiling coupled electronic and vibrational motions of chromophores in condensed phases. J. Chem. Phys. 2019, 151, 200901. [CrossRef]

21. McHale, J.L. Molecular Spectroscopy; Prentice-Hall: Upper Saddle River, NJ, USA, 1999.

22. Chen, C.; Liu, W.; Baranov, M.S.; Baleeva, N.S.; Yampolsky, I.V.; Zhu, L.; Wang, Y.; Shamir, A.; Solntsev, K.M.; Fang, C. Unveiling structural motions of a highly fluorescent superphotoacid by locking and fluorinating the GFP chromophore in solution. J. Phys. Chem. Lett. 2017, 8, 5921-5928. [CrossRef]

23. Chen, C.; Zhu, L.; Boulanger, S.A.; Baleeva, N.S.; Myasnyanko, I.N.; Baranov, M.S.; Fang, C. Ultrafast excited-state proton transfer dynamics in dihalogenated non-fluorescent and fluorescent GFP chromophores. J. Chem. Phys. 2020, 152, 021101. [CrossRef]

24. Van Stokkum, I.H.M.; Larsen, D.S.; van Grondelle, R. Global and target analysis of time-resolved spectra. Biochim. Biophys. Acta 2004, 1657, 82-104. [CrossRef]

25. Snellenburg, J.J.; Laptenok, S.P.; Seger, R.; Mullen, K.M.; van Stokkum, I.H.M. Glotaran: A Java-based graphical user interface for the R-package TIMP. J. Stat. Softw. 2012, 49, 1-22. [CrossRef]

26. Tang, L.; Zhang, S.; Zhao, Y.; Rozanov, N.D.; Zhu, L.; Wu, J.; Campbell, R.E.; Fang, C. Switching between ultrafast pathways enables a green-red emission ratiometric fluorescent-protein-based $\mathrm{Ca}^{2+}$ biosensor. Int. J. Mol. Sci. 2021, 22, 445. [CrossRef]

27. Kumpulainen, T.; Lang, B.; Rosspeintner, A.; Vauthey, E. Ultrafast elementary photochemical processes of organic molecules in liquid solution. Chem. Rev. 2017, 117, 10826-10939. [CrossRef]

28. Fang, C.; Tang, L.; Oscar, B.G.; Chen, C. Capturing structural snapshots during photochemical reactions with ultrafast Raman spectroscopy: From materials transformation to biosensor responses. J. Phys. Chem. Lett. 2018, 9, 3253-3263. [CrossRef] [PubMed] 
29. Fukushima, Y.; Iwaki, M.; Narikawa, R.; Ikeuchi, M.; Tomita, Y.; Itoh, S. Photoconversion mechanism of a green/red photosensory cyanobacteriochrome AnPixJ: Time-resolved optical spectroscopy and FTIR analysis of the AnPixJ-GAF2 domain. Biochemistry 2011, 50, 6328-6339. [CrossRef]

30. Wang, D.; Li, X.; Zhang, S.; Wang, L.; Yang, X.; Zhong, D. Revealing the origin of multiphasic dynamic behaviors in cyanobacteriochrome. Proc. Natl. Acad. Sci. USA 2020, 117, 19731-19736. [CrossRef] [PubMed]

31. Tang, L.; Wang, Y.; Zhu, L.; Lee, C.; Fang, C. Correlated molecular structural motions for photoprotection after deep-UV irradiation. J. Phys. Chem. Lett. 2018, 9, 2311-2319. [CrossRef] [PubMed]

32. Wang, D.; Qin, Y.; Zhang, M.; Li, X.; Wang, L.; Yang, X.; Zhong, D. The origin of ultrafast multiphasic dynamics in photoisomerization of bacteriophytochrome. J. Phys. Chem. Lett. 2020, 11, 5913-5919. [CrossRef]

33. Taylor, M.A.; Zhu, L.; Rozanov, N.D.; Stout, K.T.; Chen, C.; Fang, C. Delayed vibrational modulation of the solvated GFP chromophore into a conical intersection. Phys. Chem. Chem. Phys. 2019, 21, 9728-9739. [CrossRef]

34. Kim, P.W.; Freer, L.H.; Rockwell, N.C.; Martin, S.S.; Lagarias, J.C.; Larsen, D.S. Femtosecond photodynamics of the red/green cyanobacteriochrome NpR6012g4 from Nostoc punctiforme. 2. Reverse dynamics. Biochemistry 2012, 51, 619-630. [CrossRef] [PubMed]

35. Spillane, K.M.; Dasgupta, J.; Lagarias, J.C.; Mathies, R.A. Homogeneity of phytochrome Cph1 vibronic absorption revealed by resonance Raman intensity analysis. J. Am. Chem. Soc. 2009, 131, 13946-13948. [CrossRef] [PubMed]

36. Wang, D.; Qin, Y.; Zhang, S.; Wang, L.; Yang, X.; Zhong, D. Elucidating the molecular mechanism of ultrafast Pfr-state photoisomerization in bathy bacteriophytochrome PaBphP. J. Phys. Chem. Lett. 2019, 10, 6197-6201. [CrossRef]

37. Rao, A.G.; Wiebeler, C.; Sen, S.; Cerutti, D.S.; Schapiro, I. Histidine protonation controls structural heterogeneity in the cyanobacteriochrome AnPixJg2. Phys. Chem. Chem. Phys. 2021, 23, 7359-7367. [CrossRef] [PubMed]

38. Xu, X.; Höppner, A.; Wiebeler, C.; Zhao, K.-H.; Schapiro, I.; Gärtner, W. Structural elements regulating the photochromicity in a cyanobacteriochrome. Proc. Natl. Acad. Sci. USA 2020, 117, 2432-2440. [CrossRef]

39. Gepshtein, R.; Huppert, D.; Agmon, N. Deactivation mechanism of the green fluorescent chromophore. J. Phys. Chem. B 2006, 110, 4434-4442. [CrossRef] [PubMed]

40. Jung, Y.O.; Lee, J.H.; Kim, J.; Schmidt, M.; Moffat, K.; Šrajer, V.; Ihee, H. Volume-conserving trans-cis isomerization pathways in photoactive yellow protein visualized by picosecond X-ray crystallography. Nat. Chem. 2013, 5, 212-220. [CrossRef]

41. Chang, J.; Romei, M.G.; Boxer, S.G. Structural evidence of photoisomerization pathways in fluorescent proteins. J. Am. Chem. Soc. 2019, 141, 15504-15508. [CrossRef]

42. Guido, C.A.; Jacquemin, D.; Adamo, C.; Mennucci, B. On the TD-DFT accuracy in determining single and double bonds in excited-state structures of organic molecules. J. Phys. Chem. A 2010, 114, 13402-13410. [CrossRef]

43. Polyakov, I.V.; Grigorenko, B.L.; Epifanovsky, E.M.; Krylov, A.I.; Nemukhin, A.V. Potential energy landscape of the electronic states of the GFP chromophore in different protonation forms: Electronic transition energies and conical intersections. J. Chem. Theory Comput. 2010, 6, 2377-2387. [CrossRef]

44. Dasgupta, J.; Frontiera, R.R.; Taylor, K.C.; Lagarias, J.C.; Mathies, R.A. Ultrafast excited-state isomerization in phytochrome revealed by femtosecond stimulated Raman spectroscopy. Proc. Natl. Acad. Sci. USA 2009, 106, 1784-1789. [CrossRef] [PubMed]

45. Wiebeler, C.; Rao, A.G.; Gärtner, W.; Schapiro, I. The effective conjugation length is responsible for the red/green spectral tuning in the cyanobacteriochrome Slr1393g3. Angew. Chem. Int. Ed. 2019, 58, 1934-1938. [CrossRef] [PubMed]

46. Liu, Y.; Chen, Z.; Wang, X.; Cao, S.; Xu, J.; Jimenez, R.; Chen, J. Ultrafast spectroscopy of biliverdin dimethyl ester in solution: Pathways of excited-state depopulation. Phys. Chem. Chem. Phys. 2020, 22, 19903-19912. [CrossRef]

47. Fang, C.; Tang, L. Mapping structural dynamics of proteins with femtosecond stimulated Raman spectroscopy. Annu. Rev. Phys. Chem. 2020, 71, 239-265. [CrossRef]

48. Chen, C.; Zhu, L.; Fang, C. Femtosecond stimulated Raman line shapes: Dependence on resonance conditions of pump and probe pulses. Chin. J. Chem. Phys. 2018, 31, 492-502. [CrossRef]

49. Liu, W.; Tang, L.; Oscar, B.G.; Wang, Y.; Chen, C.; Fang, C. Tracking ultrafast vibrational cooling during excited state proton transfer reaction with anti-Stokes and Stokes femtosecond stimulated Raman spectroscopy. J. Phys. Chem. Lett. 2017, 8, 997-1003. [CrossRef] [PubMed]

50. Frontiera, R.R.; Shim, S.; Mathies, R.A. Origin of negative and dispersive features in anti-Stokes and resonance femtosecond stimulated Raman spectroscopy. J. Chem. Phys. 2008, 129, 064507. [CrossRef]

51. Umapathy, S.; Lakshmanna, A.; Mallick, B. Ultrafast Raman loss spectroscopy. J. Raman Spectrosc. 2009, 40, 235-237. [CrossRef]

52. Frisch, M.J.; Trucks, G.W.; Schlegel, H.B.; Scuseria, G.E.; Robb, M.A.; Cheeseman, J.R.; Scalmani, G.; Barone, V.; Petersson, G.A.; Nakatsuji, H.; et al. Gaussian 16, Revision A.03; Gaussian, Inc.: Wallingford, CT, USA, 2016.

53. Mroginski, M.A.; von Stetten, D.; Escobar, F.V.; Strauss, H.M.; Kaminski, S.; Scheerer, P.; Günther, M.; Murgida, D.H.; Schmieder, P.; Bongards, C.; et al. Chromophore structure of cyanobacterial phytochrome Cph1 in the Pr state: Reconciling structural and spectroscopic data by QM/MM calculations. Biophys. J. 2009, 96, 4153-4163. [CrossRef]

54. Wiebeler, C.; Schapiro, I. QM/MM benchmarking of cyanobacteriochrome Slr1393g3 absorption spectra. Molecules 2019, 24, 1720. [CrossRef] [PubMed]

55. Velazquez Escobar, F.; Utesch, T.; Narikawa, R.; Ikeuchi, M.; Mroginski, M.A.; Gärtner, W.; Hildebrandt, P. Photoconversion mechanism of the second GAF domain of cyanobacteriochrome AnPixJ and the cofactor structure of its green-absorbing state. Biochemistry 2013, 52, 4871-4880. [CrossRef] [PubMed] 
56. Fang, C.; Frontiera, R.R.; Tran, R.; Mathies, R.A. Mapping GFP structure evolution during proton transfer with femtosecond Raman spectroscopy. Nature 2009, 462, 200-204. [CrossRef] [PubMed]

57. Oscar, B.G.; Liu, W.; Zhao, Y.; Tang, L.; Wang, Y.; Campbell, R.E.; Fang, C. Excited-state structural dynamics of a dual-emission calmodulin-green fluorescent protein sensor for calcium ion imaging. Proc. Natl. Acad. Sci. USA 2014, 111, 10191-10196. [CrossRef] [PubMed]

58. Yang, X.; Kuk, J.; Moffat, K. Conformational differences between the Pfr and Pr states in Pseudomonas aeruginosa bacteriophytochrome. Proc. Natl. Acad. Sci. USA 2009, 106, 15639-15644. [CrossRef] [PubMed]

59. Scarbath-Evers, L.K.; Jähnigen, S.; Elgabarty, H.; Song, C.; Narikawa, R.; Matysik, J.; Sebastiani, D. Structural heterogeneity in a parent ground-state structure of AnPixJg2 revealed by theory and spectroscopy. Phys. Chem. Chem. Phys. 2017, 19, 13882-13894. [CrossRef]

60. Xu, X.-L.; Gutt, A.; Mechelke, J.; Raffelberg, S.; Tang, K.; Miao, D.; Valle, L.; Borsarelli, C.D.; Zhao, K.-H.; Gärtner, W. Combined mutagenesis and kinetics characterization of the bilin-binding GAF domain of the protein Slr1393 from the cyanobacterium Synechocystis PCC6803. ChemBioChem 2014, 15, 1190-1199. [CrossRef]

61. Kirpich, J.S.; Chang, C.-W.; Franse, J.; Yu, Q.; Escobar, F.V.; Jenkins, A.J.; Martin, S.S.; Narikawa, R.; Ames, J.B.; Lagarias, J.C.; et al. Comparison of the forward and reverse photocycle dynamics of two highly similar canonical red/green cyanobacteriochromes reveals unexpected differences. Biochemistry 2021, 60, 274-288. [CrossRef]

62. Gottlieb, S.M.; Corley, S.C.; Madsen, D.; Larsen, D.S. Note: A flexible light emitting diode-based broadband transient-absorption spectrometer. Rev. Sci. Instrum. 2012, 83, 056107. [CrossRef]

63. Liu, W.; Han, F.; Smith, C.; Fang, C. Ultrafast conformational dynamics of pyranine during excited state proton transfer in aqueous solution revealed by femtosecond stimulated Raman spectroscopy. J. Phys. Chem. B 2012, 116, 10535-10550. [CrossRef] [PubMed]

64. Zhu, L.; Liu, W.; Fang, C. A versatile femtosecond stimulated Raman spectroscopy setup with tunable pulses in the visible to near infrared. Appl. Phys. Lett. 2014, 105, 041106.

65. Merrick, J.P.; Moran, D.; Radom, L. An evaluation of harmonic vibrational frequency scale factors. J. Phys. Chem. A 2007, 111, 11683-11700. [CrossRef] [PubMed] 